\title{
Research on Dual-Channel and Dual-core \\ Plasmonic Sensor Based Photonic Crystal Fiber for Refractive Index Sensing
}

\section{Shenxi Jiao}

Northeast Electric Power University

Xiaolei Ren ( $\nabla$ r2362786162@163.com )

Northeast Electric Power University https://orcid.org/0000-0002-3813-646X

Hanrui Yang

Northeast Electric Power University

Shibo Xu

Northeast Electric Power University

\section{Research Article}

Keywords: photonic crystal fiber, surface plasmon resonance, finite element method, refractive index

Posted Date: March 11th, 2021

DOI: https://doi.org/10.21203/rs.3.rs-288469/v1

License: @ (i) This work is licensed under a Creative Commons Attribution 4.0 International License. Read Full License 


\title{
Research on dual-channel and dual-core plasmonic sensor based photonic crystal fiber for refractive index sensing
}

\author{
Shenxi Jiao ${ }^{1}$, Xiaolei Ren ${ }^{1, *}$, Hanrui Yang ${ }^{1}$,Shibo $\mathrm{Xu}^{1}$, \\ ${ }^{1}$ School of Automation Engineering, Northeast Electric Power University, Jilin 132011, China \\ *Corresponding author: r2362786162@163.com
}

\begin{abstract}
A dual-core photonic crystal fiber (PCF) with dualchannel based surface plasmon resonance (SPR) sensor is designed. The silver and gold films are severally coated in the inner walls of two large ring detection channels to excite the plasmon modes, which can make the designed sensor achieve the dual-channel sensing. The effect of structure parameters on the sensing properties and loss spectrum is numerically analyzed by finite element method (FEM). When the analyte refractive index (RI) changes from 1.340 to 1.360 , the average spectral sensitivities of 4280 and 3940 $\mathrm{nm} / \mathrm{RIU}$ are obtained for the left and right channels, corresponding to the RI resolutions of $2.34 \times 10^{-5}$ and $2.54 \times 10^{-5} \mathrm{RIU}$, respectively. The simulation results suggest that the designed dual-channel sensor can realize highly sensitive detection of two analytes simultaneously, which has a wide application in the fields of biomedical analysis and environmental monitoring.
\end{abstract}

Keywords photonic crystal fiber, surface plasmon resonance,

finite element method, refractive index

\section{Intruduction}

Under a certain incident light wavelength or frequency, the free electrons in metal interact with the incident photons and the electromagnetic waves is strongly absorbed, forming an evanescent wave with exponential attenuation on the surface of the metal and dielectric, which is called surface plasmon resonance (SPR) wave $[1,2]$. Due to the high sensitivity of SPR to the change in the refractive index (RI) of surrounding environment [3], it can be used to analyze the concentration, affinity constant and specificity caused by the absorption of the target molecules $[4,5]$. As a result, the SPR sensors have made great progress in the environmental monitoring [6], medical diagnosis [7], biotechnology [8], food safety [9] be-cause of high sensitivity, high specificity and label-free dete-ction [10,11]. Usually, SPR can be excited by traditional prism-coupled configuration based Kretschmann, but the Kretschmann structure has large volume and is not suitable for real-time and remote sensing $[12,13]$. The SPR sensors based photonics crystal fiber (PCF) have overcome above drawbacks due to small size [14], high integration and miniaturization of PCF [15]. In addition, the PCF with flexible structure design [16] and low transmission loss [17] is easy to satisfy the phase matching and achieve a strong coupling between the core mode and SPR mode [18], which makes the PCF-SPR sensors become a research hotspot.

Recently, in order to expand the application scope and satisfy the needs of reality, the researchers have done a lot of work on the structure design of PCF-SPR sensors, and the sensing characteristics have been studied by the loss spectrum method based on the mode coupling theory $[19,20]$. At present, dual-channel or multi-channel sensing has greatly potential value to master more information of the analytes, so many efforts are devoted to the relevant research. Hassani et al proposed a PCF-SPR sensor with two large semicircular channels and developed the design principles of PCF in plasmonic sensing [21]. Otupiri et al designed a SPR sensor with a small air hole introduced into the center of PCF, obtaining the RI resolutions of $5 \times 10^{-5}$ RIU for $\mathrm{HE}_{11}^{x}$ mode and $6 \times 10^{-5}$ RIU for $\mathrm{HE}_{11}^{y}$ mode in the RI range 1.33 1.34 [22]. A PCF-SPR sensor with four ring channels distributed in the PCF cladding was proposed, and utilizing four modes $\left(\mathrm{HE}_{11}^{x}, \mathrm{HE}_{11}^{y}, \mathrm{HE}_{12}^{x}\right.$, and $\left.\mathrm{HE}_{12}^{y}\right)$ achieved the wavelength sensitivities of 2200, 2400, 2200 and 2400 $\mathrm{nm} / \mathrm{RIU}$ for the RI range 1.33 1.34, respectively [23]. A PCF-SPR sensor with gold film and nanowire coated and filled separately into channel-1 and channel- 2 was proposed, which achieved the measurement of temperature and magnetic field simultaneously [24]. Additionally, a multi-channel PCF-SPR sensor with elliptical and circular air holes arranged in a hexagonal lattice was reported, and the resolutions of $5 \times 10^{-5}$ and $6 \times 10^{-5}$ RIU were verified for $\mathrm{HE}_{11}^{x}$ and $\mathrm{HE}_{11}^{y}$ modes, respectively [25]. It is more concise that using a single polarized mode on behalf of several resonance peak signals achieves the multi-channel sensing in the spectrum than that of the multiple polarized modes.

In this paper, a simple dual-channel and dual-core PCFSPR sensor is designed, and the sensing layers adopting silver and gold films are deposited in two large ring detection channels, respectively. The dual-core structure of PCF can improve the detection sensitivity and the dual-channel sensing can be realized by using different resonance characteristics of silver and gold materials. Here, a single polarized 
mode is adopted to characterize the resonance signal peaks of two channels at the same time. The results show that the average sensitivities of $4280 \mathrm{~nm} / \mathrm{RIU}$ for the left channel and $3940 \mathrm{~nm} / \mathrm{RIU}$ for the right channel are demonstrated in the wavelength interrogation.

\section{Sensing Model and Method}

The schematic of the designed dual-channel and dual-core PCF-SPR sensor is as shown in Fig. 1. It can be seen that a large air hole with a radius of $r_{1}$ is located in the model center, which is used to adjust the coupling between the core mode and SPR mode [26]. The PCF cladding consists of two layers of air holes with a radius of $r_{2}$ in a circular lattice distribution, and they have a space pitch of $\Lambda$ in the radial direction. The cladding air holes in the first layer are distributed at 60 degree intervals, and the missing air holes are used to form a dual-core structure with the light transmission. For the second layer, the cladding air holes are arranged at 30 degree intervals to limit the propagation of light in the dual-core. The silver and gold films as the sensing layers with the thickness of $d_{\mathrm{m}}$ are covered on the inner walls of two large ring channels, the left channel (Lchannel) and right channel (R-channel), which can generate two independent resonance signals utilizing different optical resonance characteristics of two materials. In addition, two metallized detection channels with a diameter of $d_{2}(1.6 \mu \mathrm{m})$ are symmetrically distributed outside the fiber structure, and have a center distance of $d_{1}(3.3 \mu \mathrm{m})$ from the central air hole, which can achieve the packaging easily and strengthen the microfluidics flow simultaneously.

Here, the background material of PCF is pure silica and the relationship between RI and free space wavelength can

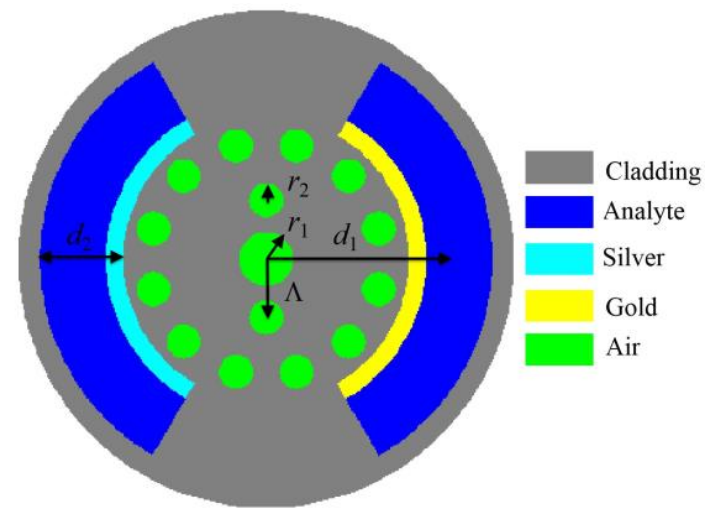

Fig. 1 Schematic of the proposed dual-channel and dual-core PCFSPR sensor

be defined by Sellmeier equation [27] :

$$
n=\sqrt{1+\sum_{i=1}^{N} \frac{\mathrm{A}_{i} \lambda^{2}}{\lambda^{2}-\mathrm{B}_{i}^{2}}}
$$

where $\lambda$ is the incident wavelength, $N=3, \mathrm{~A}_{1}=0.6961663$, $\mathrm{A}_{2}=0.4079426, \quad \mathrm{~A}_{3}=0.8974790, \quad \mathrm{~B}_{1}=0.0684043 \mu \mathrm{m}$, $\mathrm{B}_{2}=0.1162414 \mu \mathrm{m}$, and $\mathrm{B}_{3}=9.8961610 \mu \mathrm{m}$.

The L-channel and R-channel are deposited with the silver and gold films, respectively. As shown in equations (2) and
(3), the dielectric constants of silver and gold materials can be described by Drude model in visible and near-infrared bands $[28,29]$.

$$
\begin{aligned}
& \varepsilon_{\mathrm{Ag}}=1-\frac{\lambda^{2} \lambda_{\mathrm{c}}}{\lambda_{\mathrm{p}}^{2}\left(\lambda_{\mathrm{c}}+i \lambda\right)} \\
& \varepsilon_{\mathrm{Au}}=\varepsilon_{\infty}-\frac{w_{\mathrm{p}}^{2}}{w^{2}+i w w_{\tau}}
\end{aligned}
$$

where the angular frequency can be expressed as $\omega=2 \pi \mathrm{c} / \lambda$, c is the speed of light in a vacuum, $\lambda_{\mathrm{c}}=17.61400 \mu \mathrm{m}$, $\lambda_{\mathrm{p}}=0.14541 \mu \mathrm{m}, \quad \varepsilon_{\infty}=9.75, \quad w_{\mathrm{p}}=1.36 \times 10^{16} \mathrm{rad} / \mathrm{s}, \quad$ and $w_{\tau}=1.45 \times 10^{14} \mathrm{rad} / \mathrm{s}$.

When the plasmon mode is strongly coupled with the core mode, most of the energy leaks from the dual-core to metal surface and it will create a sharp loss peak in the spectrum. We can use the limiting loss of core mode to characterize the plasmon mode, which is as a function of the imaginary part of mode effective RI and can be calculated by the following equation [30]:

$$
L_{\text {oss }}(\mathrm{dB} / \mathrm{cm})=8.686 \times \frac{2 \pi}{\lambda[\mu \mathrm{m}]} \operatorname{Im}\left(n_{\text {eff }}\right) \times 10^{4}
$$

The sensitivity is used to evaluate the sensing performance of the designed sensor, which plays an important role in the detection field. When analyte RI changes, the wavelength with the resonance peak signal will create significant movement. Therefore, the spectral sensitivity in a wavelength interrogation mode can be defined by [31] :

$$
S(\mathrm{~nm} / \mathrm{RIU})=\Delta \lambda_{\text {peak }} / \Delta n_{\mathrm{a}}
$$

where $\Delta n_{\mathrm{a}}$ denotes the change in the analyte RI, and $\Delta \lambda_{\text {peak }}$ is the resonance wavelength $\mathrm{drift}$ as a response to $\Delta n_{\mathrm{a}}$. In addition, the detection resolution as another important index can be used to determine the minimum change in the analyte RI through the following equation [32] :

$$
R(\mathrm{RIU})=\Delta n_{\mathrm{a}} \cdot \Delta \lambda_{\text {min }} / \Delta \lambda_{\text {peak }}
$$

where $\Delta \lambda_{\min }$ represents the wavelength resolution of spectrometer and is assumed to be $0.1 \mathrm{~nm}$.

\section{Analysis and Discussions}

In this paper, we adopt 2D simulation environment in the COMSOL Multiphysics software based FEM to numerically analyze the mode distribution and sensing performance of the designed PCF-SPR sensor. In order to solve the required mode, a perfectly matched layer (PML) as boundary condition is considered in the simulation [33]. Firstly, when the analytes RI of L-channel $\left(n_{\mathrm{a} 1}\right)$ and R-channel $\left(n_{\mathrm{a} 2}\right)$ are set to 1.340 , the electric field distribution of the core mode at the resonance wavelengths $\left(\lambda_{\text {peak }}=528\right.$ and $\left.658 \mathrm{~nm}\right)$ is plotted in Fig. 2 and Fig. 3, respectively. The red arrows represent the polarization direction of electric field. It can be seen that there are four core modes, which are called even and odd modes for $x$-polarization and $y$-polarization, respectively. The effective RI of four core modes at the resonance wavelength $\left(\lambda_{\text {peak }}=528 \mathrm{~nm}\right)$ in Fig. 2 are 1.447521-1.421774E-5i (Fig. 2(a)), 1.447290-1.072611E-5i (Fig. 2(b)), 
1.447050-6.785469E-5i (Fig. 2(c)),

1.446784-7.680090E-5i (Fig. 2(d)), respectively. Similarly, the effective RI of four core modes at the resonance wavelength $\left(\lambda_{\text {peak }}=658 \mathrm{~nm}\right)$ in Fig. 3 are

1.436892-7.890037E-5i (Fig. 3(a)),

1.436364-6.425048E-5i (Fig. 3(b)),

1.436066-1.715782E-4i (Fig. 3(c)),

1.435465-2.068961E-4i (Fig. 3(d)), respectively. As we can see, compared to $y$-polarized core modes (Fig. 2(a) and (b), Fig. 3(a) and (b)), a small fraction of the light field is merely concentrated on the surface of silver and gold films for $x$ polarized core modes (Fig. 2(c) and (d), Fig. 3(c) and (d)), and most of the energy is confined in the dual-core, which illustrates that $y$-polarized core modes can't excite plasmon mode. In addition, it can be also found that the plasmon mode is only generated on the L-channel coated with silver film in Fig. 2(c) and (d), while the R-channel deposited with gold film has no light field. Correspondingly, the opposite result appears in Fig. 3(c) and (d). It means the independence of L-channel and R-channel and can work in two spectral regions, which provides possibility for the realization of dual-channel detection. What's more, the complex effective RI of odd mode for $x$-polarization (Fig. 2(d) and Fig. 3(d)) has a maximum value of imaginary part, which can make it more strongly coupled with SPR mode than other core modes. Therefore, the odd mode for $x$-polarization is selected as the research object for further study.
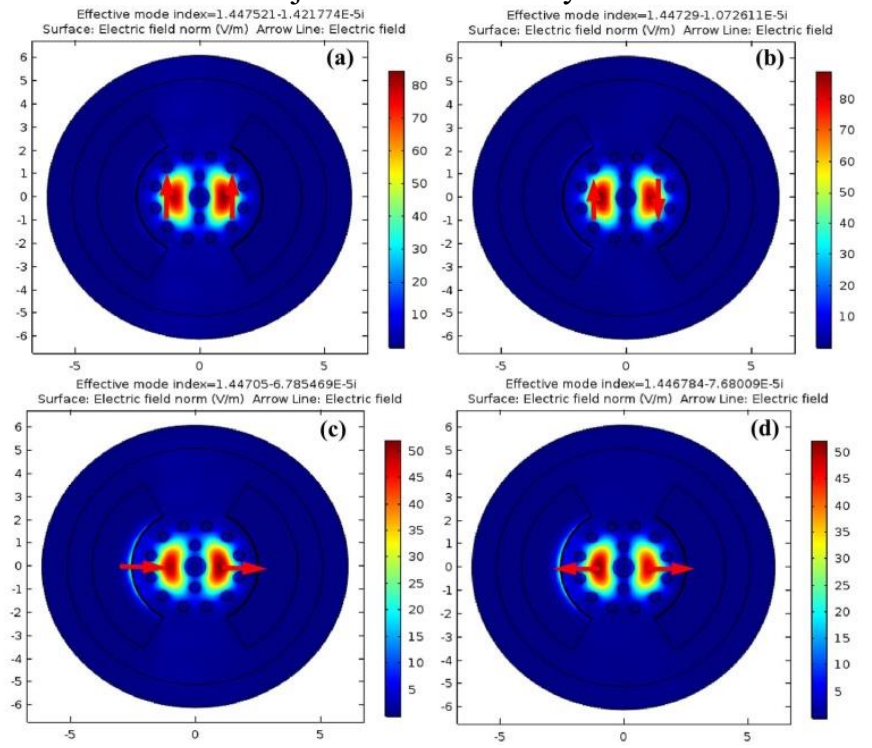

Fig. 2 Electric field distribution of the core mode. (a) even mode and (b) odd mode for $y$-polarization, (c) even mode and (d) odd mode for $x$-polarization. $\left(\Lambda=0.9 \mu \mathrm{m}, r_{1}=0.40 \mu \mathrm{m}, r_{2}=0.20 \mu \mathrm{m}\right.$, $\left.d_{\mathrm{m}}=45 \mathrm{~nm}, n_{\mathrm{a} 1}=n_{\mathrm{a} 2}=1.340, \lambda_{\text {peak }}=528 \mathrm{~nm}\right)$
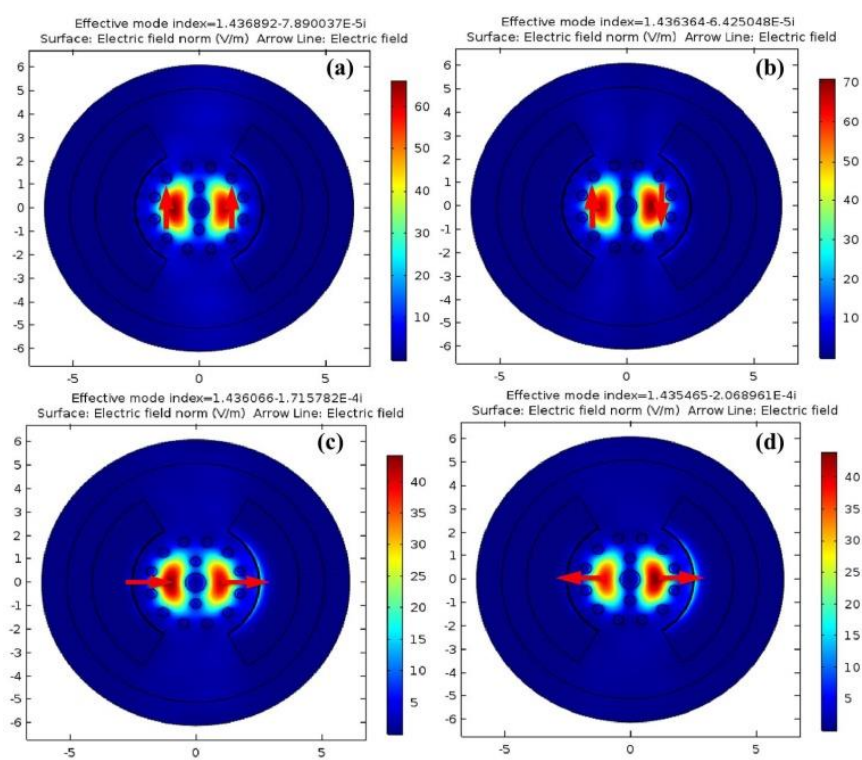

Fig. 3 Electric field distribution of the core mode. (a) even mode and (b) odd mode for $y$-polarization, (c) even mode and (d) odd mode for $x$-polarization. $\left(\Lambda=0.9 \mu \mathrm{m}, r_{1}=0.40 \mu \mathrm{m}, r_{2}=0.20 \mu \mathrm{m}\right.$, $\left.d_{\mathrm{m}}=45 \mathrm{~nm}, n_{\mathrm{a} 1}=n_{\mathrm{a} 2}=1.340, \lambda_{\text {peak }}=658 \mathrm{~nm}\right)$

Fig. 4 represents the dispersion and phase matching relationship of the core mode and SPR mode. The blue solid and dotted lines are the dispersion curves of SPR modes generated on the L-channel and R-channel, respectively. The black and red solid lines are the dispersion curve and loss spectrum of the core mode. When the real parts of effective RI of the core mode and SPR modes excited on the L-channel and Rchannel are equal, the phase matching relationships are severally satisfied at the points $(\mathrm{m})$ and $(\mathrm{n})$, which caused most of the core mode energy being strongly coupled to the surface of silver and gold films, forming two sharp resonance loss peaks at the resonance wavelengths of $528 \mathrm{~nm}$ and $658 \mathrm{~nm}$ in the spectrum. There is a wavelength interval of $130 \mathrm{~nm}$

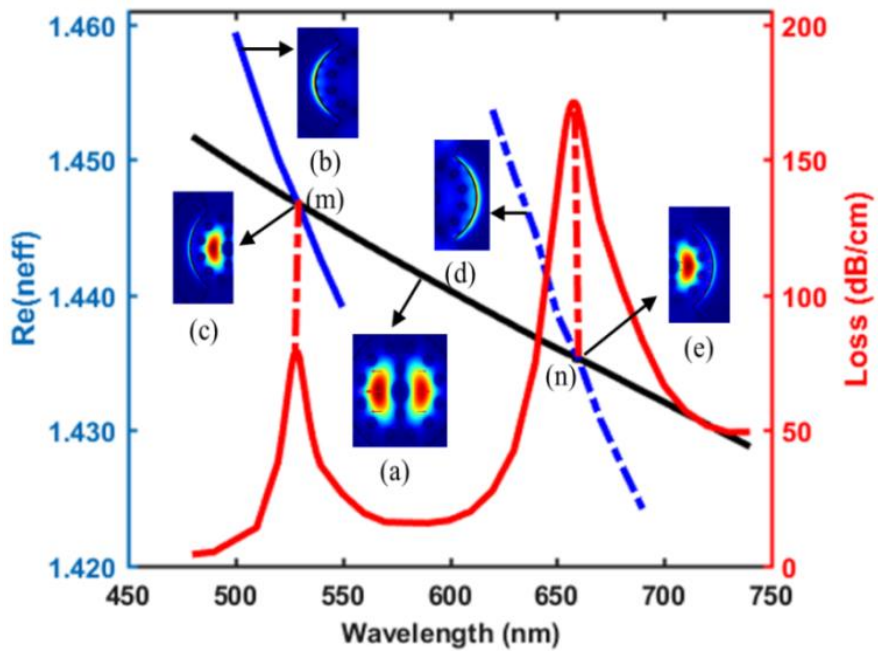

Fig. 4 Dispersion relationship of the core mode (black line) and SPR modes (blue solid and dotted lines) and loss spectrum (red line) with insets (a), (b), (c), (d) and (e) showing the electric field distribution. $\left(\Lambda=0.9 \mu \mathrm{m}, r_{1}=0.40 \mu \mathrm{m}, r_{2}=0.20 \mu \mathrm{m}, d_{\mathrm{m}}=45 \mu \mathrm{nm}\right.$, $\left.n_{\mathrm{a} 1}=n_{\mathrm{a} 2}=1.340\right)$ 
between the points $(\mathrm{m})$ and $(\mathrm{n})$, and it can provide a wide enough wavelength range to ensure the independence of $\mathrm{L}$ channel and R-channel. Inset (a) represents the energy distribution of core mode at the non-resonance wavelengths and it is principally focused in the dual-core to maintain the light transmission. Insets (b) and (d) are the light field distribution of L-channel and R-channel where the SPR modes are excited on the surfaces of silver and gold films, respectively. The electric field distribution at the resonance wavelengths (points (m) and (n)) is drawn in Insets (c) and (e), and the energy coupling between the core mode and SPR modes is observed obviously, and it is sensitive to the changes in the analytes RI near the metal surface caused by the molecular absorption, which can achieve dual-channel sensing through a single optical fiber mode in the composite spectrum.

As the sensing layers, the influence of metal film thickness on the half width and intensity of the resonance peak has been systematically studied [34,35]. Fig. 5 shows the effect of the thickness of silver and gold films $\left(d_{\mathrm{m}}\right)$ on the loss spectrum. It can be seen that the resonance wavelength of L-channel increases from 518 to $536 \mathrm{~nm}$, and the resonance intensity decreases from 120.3801 to $51.5931 \mathrm{~dB} / \mathrm{cm}$ with the increase of $d_{\mathrm{m}}$ from 40 to $50 \mathrm{~nm}$. It has the same change trend for $\mathrm{R}$-channel, which is that the resonance wavelength enlarges from 644 to $670 \mathrm{~nm}$ and the resonance strength reduces from 253.2790 to $133.4043 \mathrm{~dB} / \mathrm{cm}$ in the calculated range. The reason is that the incident light will be strongly absorbed by the silver and gold films with large imaginary parts, resulting in less energy penetrating the metal surface. with $d_{\mathrm{m}}$ increasing. In addition, the half width of resonance peak and loss at the off-resonance wavelength also expand [36].

The effect of space pitch $(\Lambda)$ on the loss spectrum of the core mode is shown in Fig. 6 . When $\Lambda$ changes from 0.8 to $1.0 \mu \mathrm{m}$, the resonance wavelength of L-channel blueshifts from 566 to $513 \mathrm{~nm}$, and the R-channel moves towards a short wavelength from 701 to $633 \mathrm{~nm}$. While the resonance loss shows the same trend of decrease first and then increase for two channels, there are minimum values of 79.7665 $\mathrm{dB} / \mathrm{cm}$ for L-channel and $171.4112 \mathrm{~dB} / \mathrm{cm}$ for R-channel

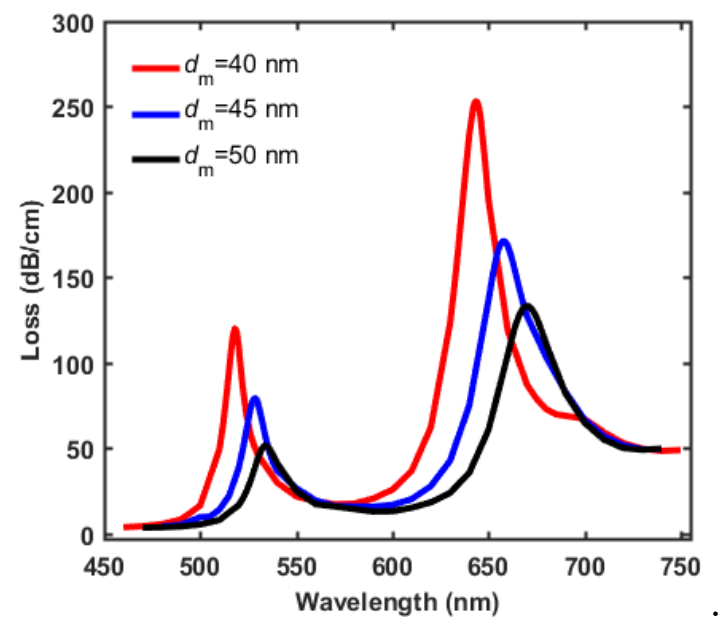

Fig. 5 Loss spectrum of the core mode for different $d_{\mathrm{m}}$ from 40 to $50 \mathrm{~nm} .\left(\Lambda=0.9 \mu \mathrm{m}, r_{1}=0.40 \mu \mathrm{m}, r_{2}=0.20 \mu \mathrm{m}, n_{\mathrm{a} 1}=n_{\mathrm{a} 2}=1.340\right)$

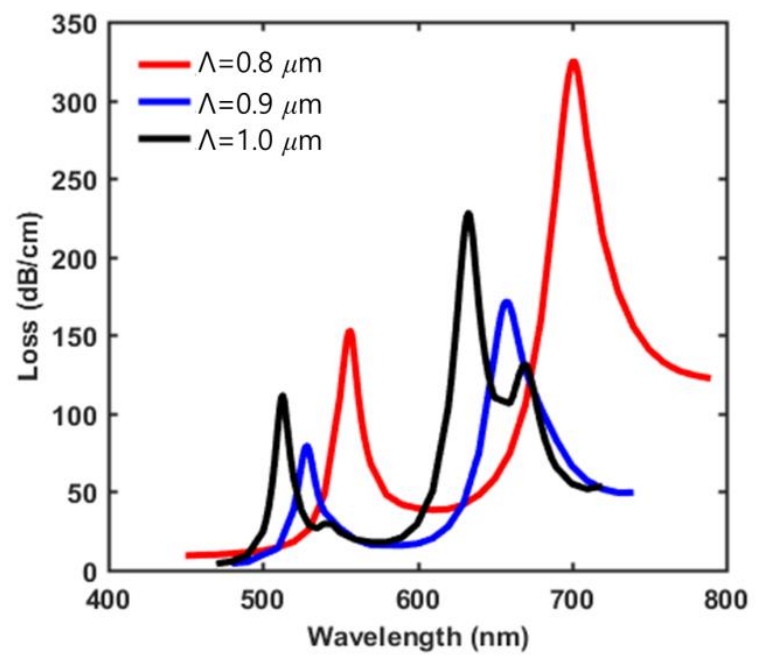

Fig. 6 Loss spectrum of the core mode for different $\Lambda$ from 0.8 to $1.0 \mu \mathrm{m} .\left(r_{1}=0.40 \mu \mathrm{m}, r_{2}=0.20 \mu \mathrm{m}, d_{\mathrm{m}}=45 \mathrm{~nm}, n_{\mathrm{a} 1}=n_{\mathrm{a} 2}=1.340\right)$

when $\Lambda$ is $0.9 \mu \mathrm{m}$, which can reduce the light transmission loss and promote the coupling between the core mode and SPR modes better. In addition, there is a small peak close to the main peak of R-channel for $\Lambda=1.0 \mu \mathrm{m}$, which makes the spectrum become untidy and is adverse to the dual-channel sensing.

The influence of the air holes radius of the PCF center $\left(r_{1}\right)$ and cladding $\left(r_{2}\right)$ on the sensing properties of the designed
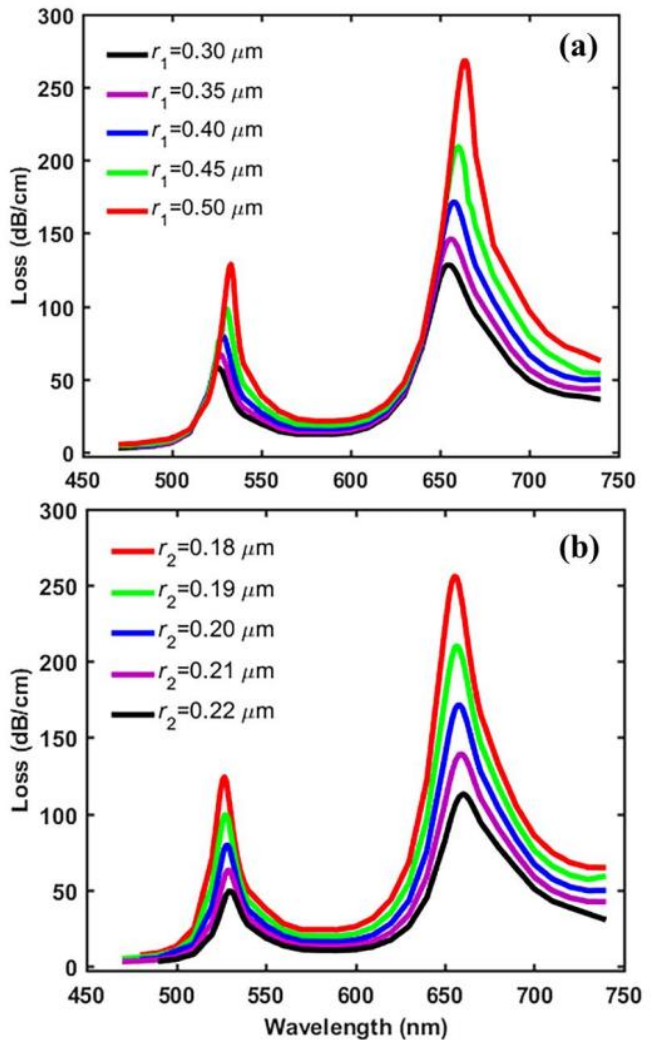

Fig. 7 (a) Loss spectrum of the core mode for different $r_{1}$ from 0.30 to $0.50 \mu \mathrm{m}$ and (b) different $r_{2}$ from 0.18 to $0.22 \mu \mathrm{m}$. $(\Lambda=0.9 \mu \mathrm{m}$, $\left.d_{\mathrm{m}}=45 \mathrm{~nm}, n_{\mathrm{a} 1}=n_{\mathrm{a} 2}=1.340\right)$ 
sensor is simulated and discussed. Fig. 7(a) shows the loss spectrum of the core mode for $r_{1}$ varying from 0.30 to 0.50 $\mu \mathrm{m}$ with a step of $0.05 \mu \mathrm{m}$. It can be seen that the resonance wavelength is tuned in the range from 526 to $533 \mathrm{~nm}$ with a variation of $7 \mathrm{~nm}$ for L-channel and the range from 654 to $664 \mathrm{~nm}$ with a variation of $10 \mathrm{~nm}$ for R-channel, corresponding to the resonance peak changing from 58.0115 to $128.8000 \mathrm{~dB} / \mathrm{cm}$ and 128.8270 to $268.9466 \mathrm{~dB} / \mathrm{cm}$, respectively. Likewise, the effect of $r_{2}$ on the loss spectrum of the core mode is studied. As shown in Fig. 7(b), the resonance wavelength remains almost unchanged for two channels, while the resonance intensity decreases from 124.1700 to $49.5493 \mathrm{~dB} / \mathrm{cm}$ for L-channel and diminishes from 255.9930 to $112.7286 \mathrm{~dB} / \mathrm{cm}$ for R-channel as $r_{2}$ increases from 0.18 to $0.22 \mu \mathrm{m}$ with a step of $0.01 \mu \mathrm{m}$. It can be explained by the fact that a large air hole located in the center can lower the effective RI of core mode [37], leading to the RI difference of the core mode and cladding mode reducing, which causes more energy leakage from the dual-core to metal surfaces. On the contrary, the equivalent RI of the PCF cladding decreases with the increase of $r_{2}$, corresponding to the RI difference enlarging, resulting in more energy being confined in the dual-core. Therefore, it provides a method to regulate the position of resonance peak by changing $r_{1}$ and $r_{2}$.

The detection sensitivity of the designed dual-channel PCF-SPR sensor is evaluated. When the RI of L-channel
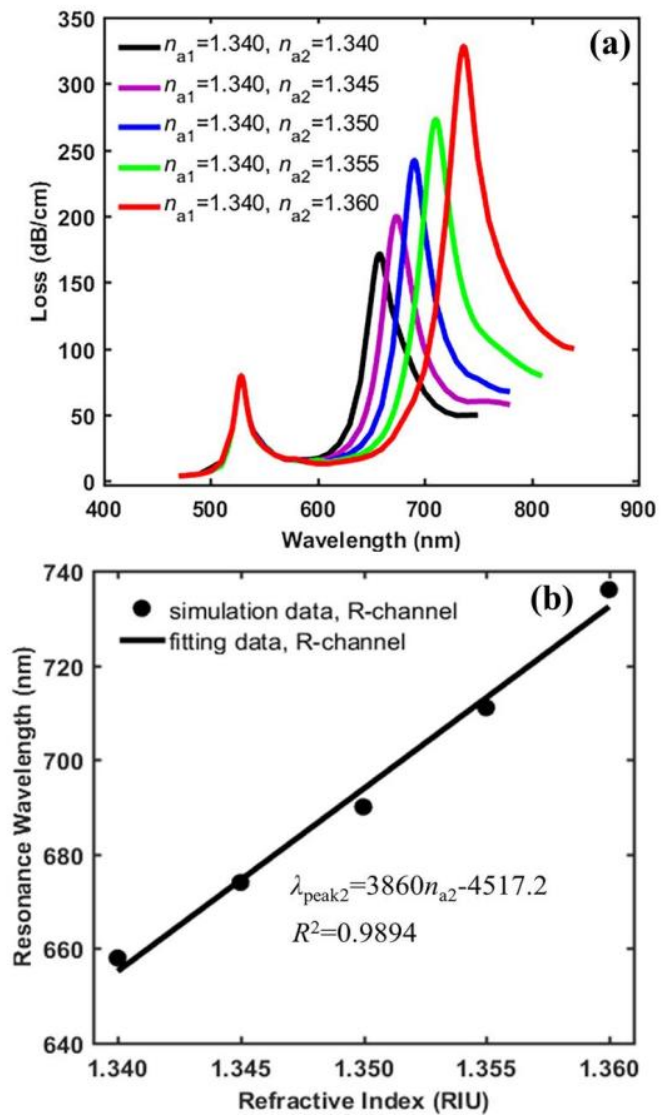

Fig. 8 (a) Loss spectrum of the core mode for different $n_{\mathrm{a} 2}$ from 1.340 to 1.360 . (b) Linear fitting line of $\lambda_{\text {peak2 }}$ with respect to $n_{\mathrm{a} 2}$. $\left(\Lambda=0.9 \mu \mathrm{m}, r_{1}=0.40 \mu \mathrm{m}, r_{2}=0.20 \mu \mathrm{m}, d_{\mathrm{m}}=45 \mathrm{~nm}, n_{\mathrm{a} 1}=1.340\right)$ $\left(n_{\mathrm{a} 1}\right)$ is fixed as 1.340 and the RI of R-channel $\left(n_{\mathrm{a} 2}\right)$ varies from 1.340 to 1.360 with a step of 0.005 , the loss spectrum of the core mode is drawn in Fig. 8 (a). As we can see, the

$$
\lambda_{\text {peak } 2}=3860 n_{\mathrm{a} 2}-4517.2 \quad\left(R^{2}=0.9894\right)
$$

resonance wavelength redshifts from 658 to $736 \mathrm{~nm}$ with the increase of $n_{\mathrm{a} 2}$ for the R-channel, and without change for the L-channel, which illustrates that the L-channel and Rchannel are independent channels and the R-channel can be used as a sensing channel separately. Fig. 8(b) shows the linear fitting line of the resonance wavelength of R-channel $\left(\lambda_{\text {peak2 }}\right)$ in relation to $n_{\mathrm{a} 2}$. The fitting relationship with a linearity of $R^{2}=0.9894$ is expressed by equation (7). The coefficient of first order is on behalf of an average spectral sensitivity of $3860 \mathrm{~nm} / \mathrm{RIU}$ for R-channel in the $n_{\mathrm{a} 2}$ range of $1.340 \sim 1.360$. At the same time, a RI resolution of $2.59 \times 10^{-5}$ RIU is also obtained according to equation (6).

Similarly, Fig. 9(a) shows the loss spectrum of the core mode when $n_{\mathrm{a} 2}=1.340$ and $n_{\mathrm{a} 1}$ changes from 1.340 to 1.360 with a step of 0.005 . It can be seen that $\lambda_{\text {peak } 1}$ for L-channel enlarges from 528 to $614 \mathrm{~nm}$ with the increase of $n_{\mathrm{a} 1}$, and $\lambda_{\text {peak2 }}$ for R-channel is unchanged. When $n_{\mathrm{a} 1}$ is greater than 1.360 , the resonance signals carried by $\lambda_{\text {peak } 1}$ and $\lambda_{\text {peak } 2}$ will create the overlap, which is no longer appropriate for Lchannel as a separate sensing channel to complete the detection. The linear fitting line of $\lambda_{\text {peak } 1}$ with respect to $n_{\mathrm{a} 1}$ is plotted in Fig. 9(b). The fitting relationship with a better linearity of $\mathrm{R}^{2}=0.9937$ is expressed by equation (8). As a
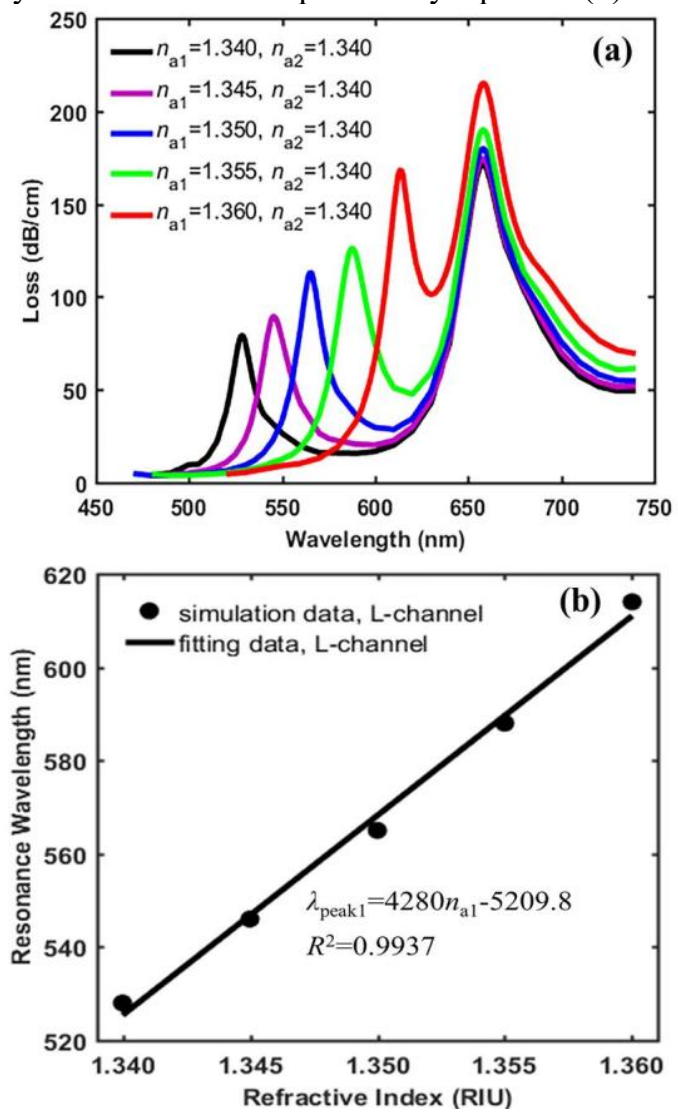

Fig. 9 (a) Loss spectrum of the core mode for different $n_{\mathrm{a} 1}$ from 1.340 to 1.360 . (b) Linear fitting line of $\lambda_{\text {peak } 1}$ with respect to $n_{\mathrm{a} 1}$. $\left(\Lambda=0.9 \mu \mathrm{m}, r_{1}=0.40 \mu \mathrm{m}, r_{2}=0.20 \mu \mathrm{m}, d_{\mathrm{m}}=45 \mathrm{~nm}, n_{\mathrm{a} 2}=1.340\right)$ 
consequence, an average spectral sensitivity of $4280 \mathrm{~nm} / \mathrm{RIU}$ for L-channel is obtained in the $n_{\mathrm{a} 1}$ range of $1.340 \sim 1.360$, corresponding to a RI resolution of $2.34 \times 10^{-5} \mathrm{RIU}$.
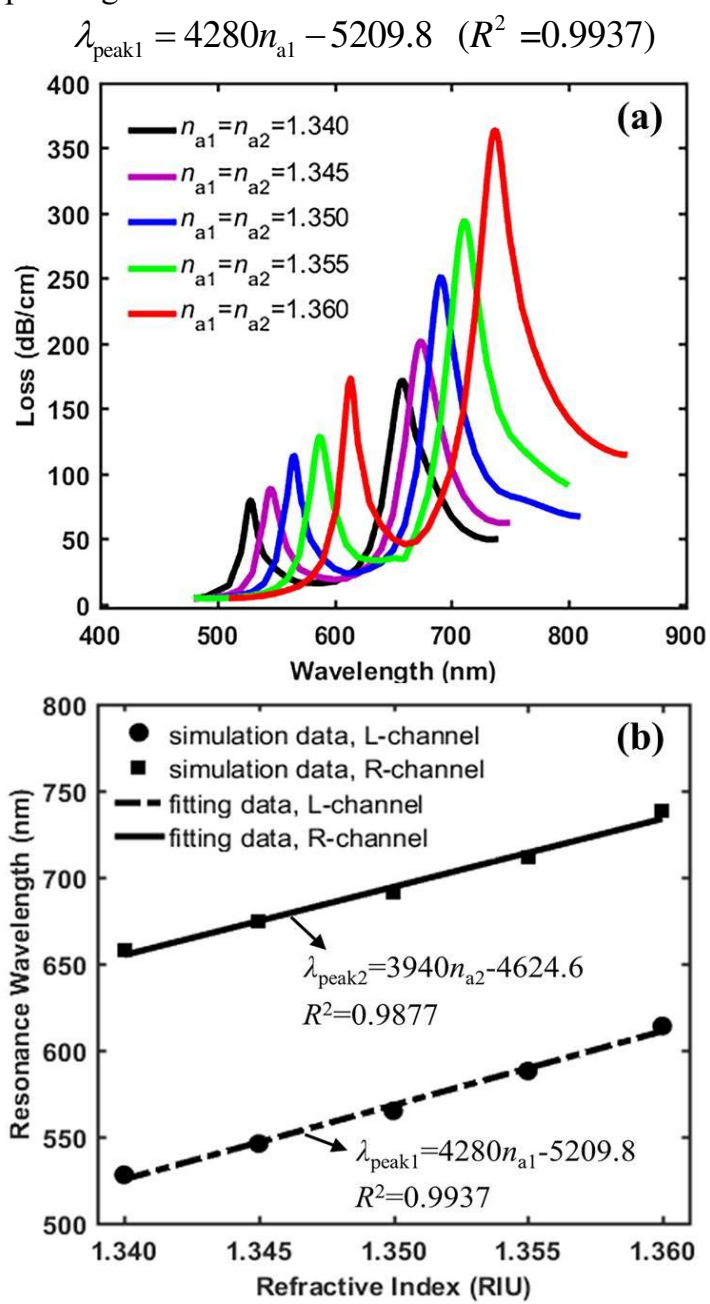

Fig.10 (a) Loss spectrum of the core mode for different $n_{\mathrm{a} 1}$ and $n_{\mathrm{a} 2}$ from 1.340 to 1.360 . (b) Linear fitting curves of $\lambda_{\text {peak } 1}$ and $\lambda_{\text {peak } 2}$ with respect to $n_{\mathrm{a} 1}$ and $n_{\mathrm{a} 2}$, respectively. $\left(\Lambda=0.9 \mu \mathrm{m}, r_{1}=0.40 \mu \mathrm{m}\right.$, $\left.r_{2}=0.20 \mu \mathrm{m}, d_{\mathrm{m}}=45 \mathrm{~nm}\right)$

Through the above analysis (Fig. 8 and Fig. 9), when the analyte RI of L-channel or R-channel is fixed, both can serve as independent detection channels and can be used alone. During the simultaneous operation mode of L-channel and R-channel, the loss spectrum of the core mode is as shown in Fig. 10 (a) for $n_{\mathrm{a} 1}$ and $n_{\mathrm{a} 2}$ varying from 1.340 to 1.360 with a step of 0.005 . The resonance wavelengths of two channels ( $\lambda_{\text {peak1 }}$ and $\left.\lambda_{\text {peak } 2}\right)$ both move towards long wavelengths, corresponding to the changes of $\lambda_{\text {peak } 1}$ from 528 to $614 \mathrm{~nm}$ and $\lambda_{\text {peak } 2}$ from 658 to $738 \mathrm{~nm}$. They are almost identical to the values given in Fig. 8 and Fig. 9, which verifies the independence of two channels again. In addition, the linear fitting curves of $\lambda_{\text {peak } 1}$ and $\lambda_{\text {peak } 2}$ with respect to $n_{\mathrm{a} 1}$ and $n_{\mathrm{a} 2}$ are simultaneously plotted in Fig. 10(b) and the fitt-ing relationships are shown in equation (9). In the operation mode of dual-channel, the wavelength sensitivities of 4280 $\mathrm{nm} / \mathrm{RIU}$ with a linearity of $\mathrm{R}^{2}=0.9937$ and $3940 \mathrm{~nm} / \mathrm{RIU}$ with a linearity of $R^{2}=0.9877$ are obtained in the RI sensing range between 1.340 and 1.360 , corresponding to the RI resolutions of $2.34 \times 10^{-5}$ and $2.54 \times 10^{-5}$ RIU, respectively, which can achieve better sensing performance than that of Ref [22], [23] and [25].

$$
\begin{cases}\lambda_{\text {peak } 1}=4280 n_{\mathrm{a} 1}-5209.8 & \left(R^{2}=0.9937\right) \\ \lambda_{\text {peak } 2}=3940 n_{\mathrm{a} 2}-4624.6 & \left(R^{2}=0.9877\right)\end{cases}
$$

\section{Conclusion}

A dual-core PCF-SPR sensor with dual-channel separately covered with silver and gold films is designed. Utilizing different optical resonance properties of the silver and gold materials, the proposed sensor can operate in two modes: single channel and dual-channel sensing. The odd mode for $\mathrm{x}$-polarization is selected as the research object through the coupling properties analysis by FEM, which can simultaneously characterize two resonance signal peaks. In the operation mode of single channel detection, the spectral sensitivities of $4280 \mathrm{~nm} / \mathrm{RIU}$ for L-channel and 3860 $\mathrm{nm} / \mathrm{RIU}$ for R-channel are obtained. At the same time, it also achieves the wavelength sensitivities of $4280 \mathrm{~nm} / \mathrm{RIU}$ for Lchannel and $3940 \mathrm{~nm} / \mathrm{RIU}$ for R-channel in the operation mode of dual-channel sensing when analytes RI changes between 1.340 and 1.360, which can be used for biochemical detection and environmental monitoring in the multicomponent analysis.

\section{Declarations}

Ethical Approval All authors declare that this manuscript is original, has not been published before and is not currently being considered for publication elsewhere. We understand that the Corresponding Author is the sole contact for the Editorial process.

Consent to Participate We confirm that the manuscript has been read and approved by all named authors and that there are no other persons who satisfied the criteria for authorship but are not listed. We further confirm that the order of authors listed in the manuscript has been approved by all of us. All authors read and approved the final manuscript.

Consent to Publish We would like to draw the attention of the editor to the following publications of one or more of us that refer to aspects of the manuscript presently being submitted. Where relevant copies of such publications are attached. We further confirm that the order of authors listed in the manuscript has been approved by all of us.

Authors Contributions All authors contributed to the study conception and design. Material preparation, data collection and analysis were performed by Shengxi Jiao and Xiaolei Ren. The first draft of the manuscript was written by Xiaolei Ren. Data curation and visualization by Hanrui Yang, Shibo Xu and all authors commented on previous versions of the manuscript.

Funding No funds, grants, or other support was received.

Competing Interests The authors declare no conflicts of interest.

Availability of data and materials All data, models, and code generated or used during the study appear in the submitted manuscript.

\section{References}

1. K. Matsubara, S. Kawata, and S. Minami (1988) Optical chemical sensor based on surface plasmon measuremen. Appl. Opt. 27(6):1160-1163.

2. G. W. An, S. G. Li, W. Qin, W. Zhang, Z. K. Fan, and Y. J. Bao (2014) High-sensitivity refractive index sensor based on 
D-shaped photonic crystal fiber with rectangular lattice and nanoscale gold film. Plasmonics. 9(6):1355-1360.

3. P. B. Bing, S. C. Huang, J. L. Sui, H. Wang, and Z. Y. Wang (2018) Analysis and improvement of a dual-core photonic crystal fiber sensor. Sensors. 18(7):2051-2059.

4. A. G. Brolo (2012). Plasmonics for future biosensors. Nat. Photonics. 6(11):709-713

5. M. A. Otte, B. Sepúlveda, W. H. Ni, J. P. Juste, L. M. LizMarzán, and L. M. Lechuga (2010) Identification of the optimal spectral region for plasmonic and nanoplasmonic sensing. ACS Nano. 4(1):349-357.

6. C. Mouvet, R. D. Harris, C. Maciag, B. J. Luff, J. S. Wilkinson, J. Piehler, A. Brccht, G. Gauglitz, R. Abukncsha, and G. Ismail (1997) Determination of simazine in water samples by waveguide surface plasmon resonance. Anal. Chim. Acta. 338(2):109-117.

7. J. Homola (2008) Surface plasmon resonance sensors for detection of chemical and biological species. Chem. Rev. 108(2):462-493.

8. J. N. Anker, W. P. Hall, O. Lyandres, N. C. Shah, J. Zhao, and R. P. Van Duyne (2008) Biosensing with plasmonic nanosensors. Nat. Mater. 7(6):442-453.

9. M. Piliarik, L. Párová, and J. Homola (2009) High-throughput SPR sensor for food safety. Biosens. Bioelectron. 24(5):13991404.

10. Z. Yang, L. Xia, C. Li, X. Chen, and D. M. Liu (2019) A surface plasmon resonance sensor based on concave-shaped photonic crystal fiber for low refractive index detection. Opt. Commun. 430:195-203.

11. A. Hassani, and M. Skorobogatiy (2009) Photonic crystal fiber-based plasmonic sensors for the detection of biolayer thickness. J. Opt. Soc. Am. B. 26(8):1550-1557.

12. X. Yu, S. Y. Zhang, Y. Zhang, H. P. Ho, P. Shum, H. R. Liu, D. M. Liu (2010) An efficient approach for investigating surface plasmon resonance in asymmetric optical fibers based on birefringence analysis. Opt. Express. 18(17): 17950-17957.

13. J. N. Dash and R. Jha (2014) SPR biosensor based on polymer PCF coated with conducting metal oxide. IEEE Photonic. Tech. L. 26(6):595-598.

14. J. N. Dash, and R. Jha (2014) Graphene-based birefringent photonic crystal fiber sensor using surface plasmon resonance. IEEE Photonic. Tech. L. 26(11):1092-1095.

15. M. F. O. Hameed, Y. K. A. Alrayk, and S. S. A. Obayya (2016) Self-calibration highly sensitive photonic crystal fiber biosensor. IEEE Photonics. J. 8(3):6802912.

16. A. A. Rifat, R. Ahmed, G. A. Mahdiraji, and F. R. M. Adikan (2017) Highly sensitive D-shaped photonic crystal fiber-based plasmonic biosensor in visible to near-IR. IEEE Sens. J. 17:2776-2783.

17. M. N. Hossen, Md. Ferdous, Md. A. Khalek, S. Chakma, B. K. Paul, and K. Ahmed (2018) Design and analysis of biosensor based on surface plasmon resonance. Sensing and Bio-Sensing Research. 21:1-6.

18. J. J. Wu, S. G. Li, X. Y. Wang, M Shi, X. X. Feng, and Y. D. Liu (2018) Ultrahigh sensitivity refractive index sensor of a Dshaped PCF based on surface plasmon resonance. Appl. Opt. 57(15):4002-4007.

19. F. K. Shi, G. Y. Zhou, D. M. Li, L. Peng, Z. Y. Hou, and C. M. Xia (2015) Surface plasmon mode coupling in photonic crystal fiber symmetrically filled with $\mathrm{Ag} / \mathrm{Au}$ alloy wires. Plasmonics 10(2):335-340.

20. N. N. Luan, R. Wang, W. H. Lv, and J. Q. Yao (2015) Surface plasmon resonance sensor based on D-shaped microstructured optical fiber with hollow core. Opt. Express. 23(7):8576-8582.
21. A. Hassani, and M. Skorobogatiy (2006) Design of the microstruc-tured optical fiber-based surface plasmon resonance sensors with enhanced microfluidics. Opt. Express. 14(24):11616-11621.

22. R. Otupiri, E. K. Akowuah, S. Haxha, H. Ademgil, F. Abdelmalek, and A. Aggoun (2014) A novel birefrigent photonic crystal fiber surface plasmon resonance biosensor. IEEE Photonics. J. 6(4):1-11.

23. S. I. Azzam, M. F. O. Hameed, R. E. A. Shehata, A. M. Heikal, and S. S. A. Obayya (2016) Multichannel photonic crystal fiber surface plasmon resonance based sensor. Opt Quant Electron. 48(2):142-152.

24. H. Liu, Y. Wang, S. Q. Wei, C. H. Zhu, C. Tan, M. Wang, and D. Q. Cheng (2017) Simultaneous dual-parameter measurement based on dual-channel surface plasmon resonance in photonic crystal fiber. Optik. 145:582-588.

25. E. K. Akowuah, T. Gorman, H. Ademgil, S. Haxha, G. K. Robinson, and J. V. Oliver (2012) Numerical analysis of a photonic crystal fiber for biosensing applications. IEEE J. Quantum. Elect. 48(11):1403-1410.

26. A. Hassani, and M. Skorobogatiy (2007) Design criteria for micros-tructured optical fiber based surface-plasmonresonance sensors. J. Opt. Soc. Am. B. 24(6):1423-1429.

27. R. K. Gangwar, and V. K. Singh (2017) Highly sensitive surface plasmon resonance based D-shaped photonic crystal fiber refractive index sensor. Plasmonics. 12(5):1367-1372.

28. S. X. Jiao, S. F. Gu, H. R. Yang, H. R. Fang, and S. B. Xu (2018) Highly sensitive dual-core photonic crystal fiber based on surface plasmon resonance sensor with silver nanocontinuous grating. Appl. Opt. 57(28):8350-8358.

29. A. A. Rifat, F. Haider, R. Ahmed, G. A. Mahdiraji, F. R. M. Adikan, and A. E. Miroshnichenko (2018) Highly sensitive selec-tively coated photonic crystal fiber-based plasmonic sensor. Opt. Lett. 43(4):891-894.

30. T. S. Wu, Y. Shao, Y. Wang, S. Q. Cao, W. P. Cao, F. Zhang, C. R. Liao, J. He, Y. J. Huang, M. X. Hou, and Y. P. Wang (2017) Surface plasmon resonance biosensor based on goldcoated side-polished hexagonal structure photonic crystal fiber. Opt. Express. 25(17):20313-20322.

31. J. J. Lu, Y. Li, Y. H. Han, Y. Liu, and J. M. Gao (2018) Dshaped photonic crystal fiber plasmonic refractive index sensor based on gold grating. Appl. Opt. 57(19):5268-5272.

32. A. A. Rifat, G. A. Mahdiraji, Y. M. Sua, R. Ahmed,3 Y. G. Shee, and F. R. M. Adikan (2016) Highly sensitive multi-core flat fiber surface plasmon resonance refractive index sensor. Opt. Express. 24(3):2485-2495.

33. M. R. Momota, and M. R. Hasan (2018) Hollow-core silver coated photonic crystal fiber plasmonic sensor. Opt. Mater. 76:287-294.

34. F. N. Zha, J. S Li, P. J. Sun, H. J. Ma (2019) Highly sensitive selectively coated D-shape photonic crystal fibers for surface plasmon resonance sensing. Phys. Lett. A. 383(15):1825-1830.

35. C. Liu, L Yang, X. L. Lu, Q. Liu, F. M. Wang, J. W. Lv, T. Sun, H. W. Mu, and P. K. Chu (2017) Mid-infrared surface plasmon resonance sensor based on photonic crystal fibers. Opt. Express. 25(13):14227-14237.

36. C. Liu, W. Q. Su, F. M. Wang, X. L. Li, L. Yang, T. Sun, H W. Mu, and P. K. Chu (2019) Theoretical assessment of a highly sensitive photonic crystal fibre based on surface plasmon resonance sensor operating in the near-infrared wavelength. J. Mod. Optic. 66(1):1-6.

37. A. H. El-Saeed, A. E. Khalil, M. F. O. Hameed, M. Y. Azab, and S. S. A. Obayya (2019) Highly sensitive SPR PCF biosensors based on $\mathrm{Ag} / \mathrm{TiN}$ and $\mathrm{Ag} / \mathrm{ZrN}$ configurations. Opt. Quant. Electron. 51(2):56-73. 
Figures

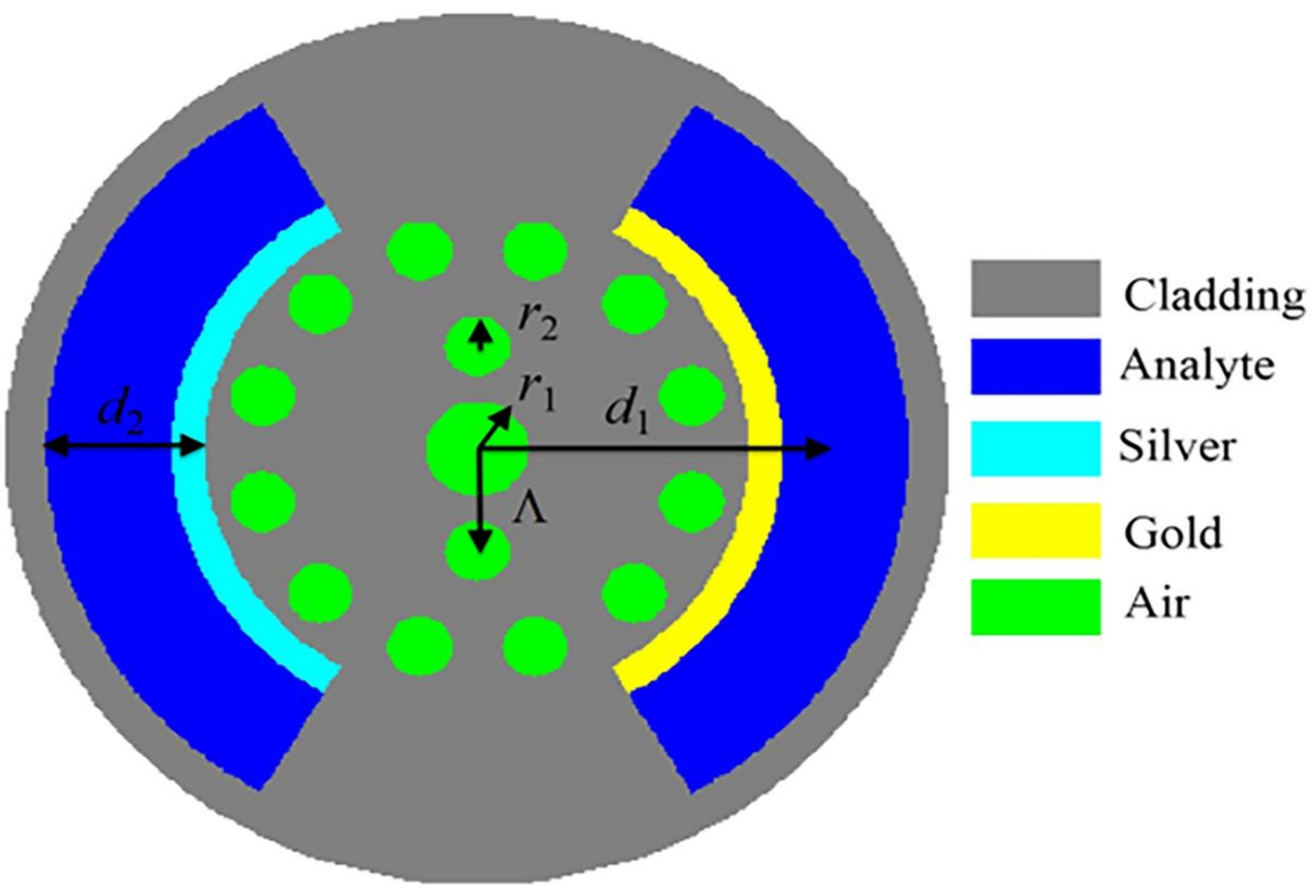

Figure 1

Schematic of the proposed dual-channel and dual-core PCF-SPR sensor 
Effective mode index $=1,447521-1,421774 \mathrm{E}-5 \mathrm{i}$ Surface: Electric field norm (V/m) Arrow Line: Electric field

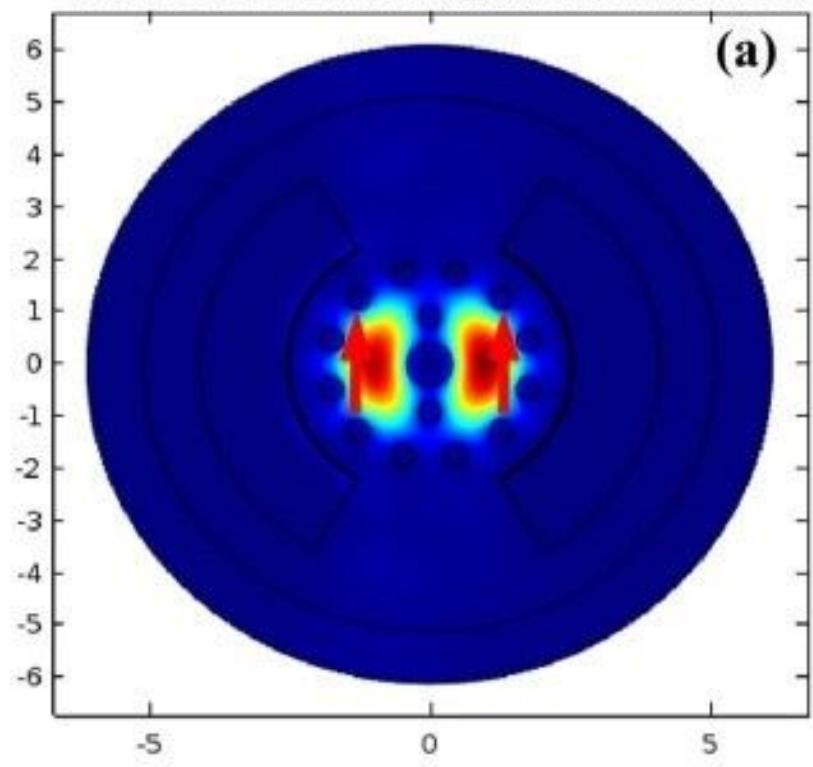

Effective mode index $=1.44705-6.785469 \mathrm{E}-5 \mathrm{i}$ Surface: Electric field norm (V/m) Arrow Line: Electric field

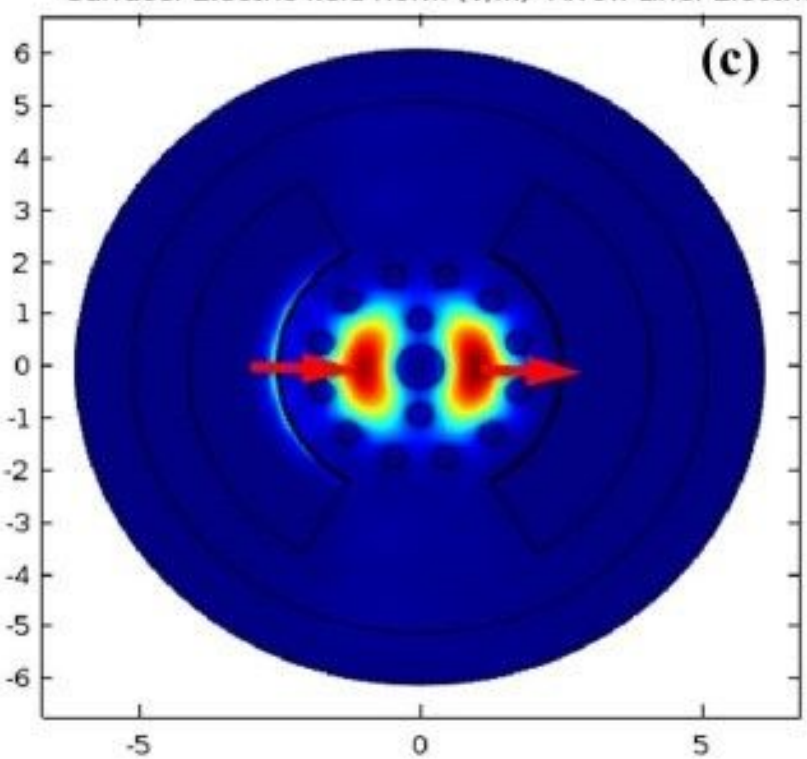

Effective mode index $=1,44729-1.072611 \mathrm{E}-5 \mathrm{i}$ Surface: Electric field norm (V/m) Arrow Line: Electric field

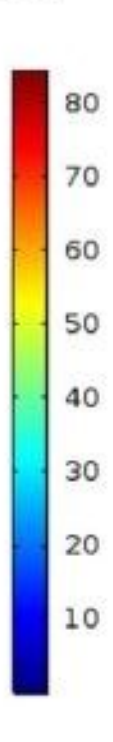
6
5
4
3
2
1
0
-1
-2
-3
-4
-5
-6

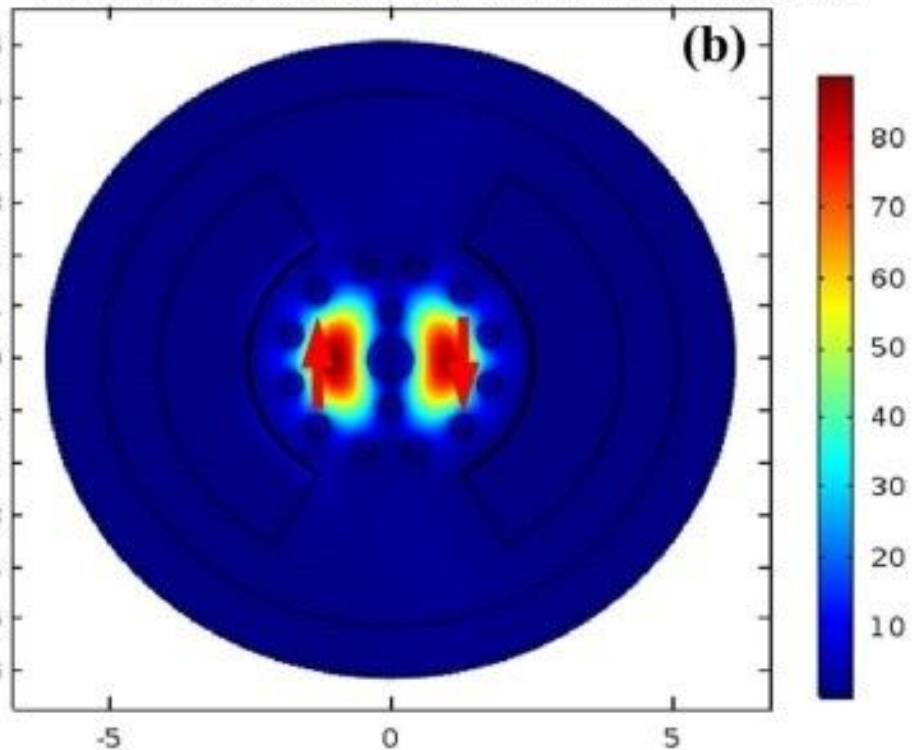

Effective mode index $=1.446784-7.68009 E-5 i$ Surface: Electric field norm (V/m) Arrow Line: Electric field

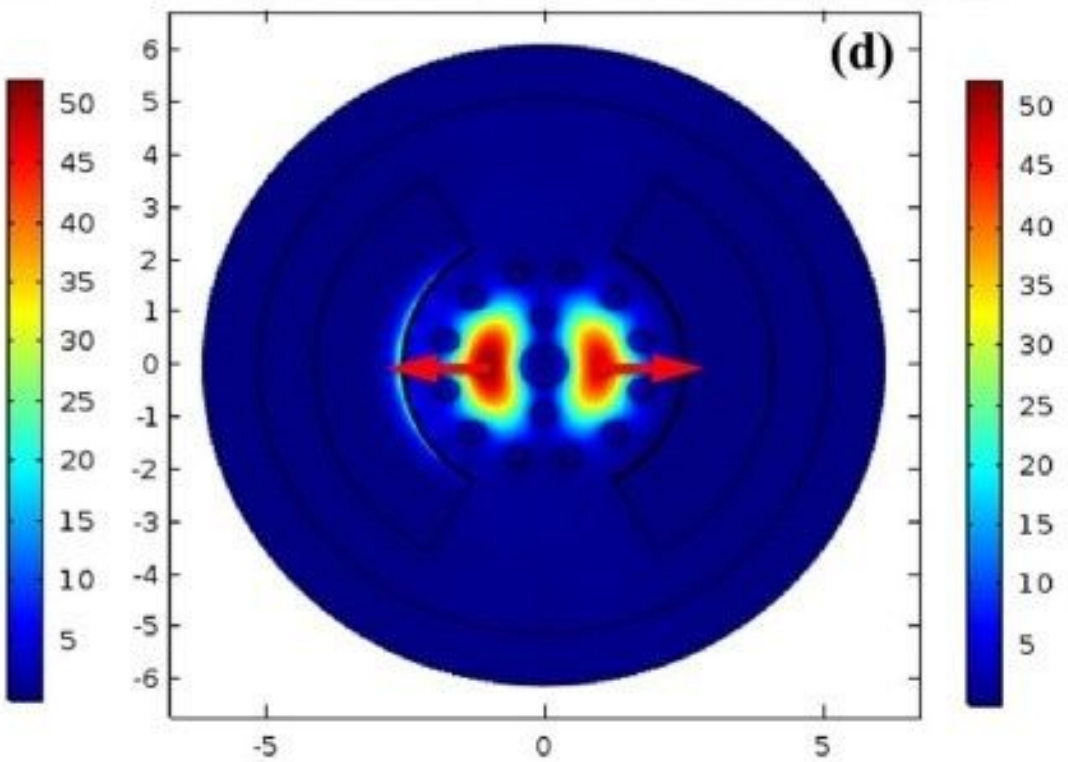

Figure 2

Electric field distribution of the core mode. (a) even mode and (b) odd mode for y-polarization, (c) even mode and (d) odd mode for $x$-polarization. $(\Lambda=0.9 \mu \mathrm{m}, \mathrm{r} 1=0.40 \mu \mathrm{m}, \mathrm{r} 2=0.20 \mu \mathrm{m}, \mathrm{dm}=45 \mathrm{~nm}$, na1=na2=1.340, $\lambda$ peak=528 nm) 
Effective mode index $=1.436892-7.890037 \mathrm{E}-5 \mathrm{i}$

Surface: Electric field norm (V/m) Arrow Line: Electric field

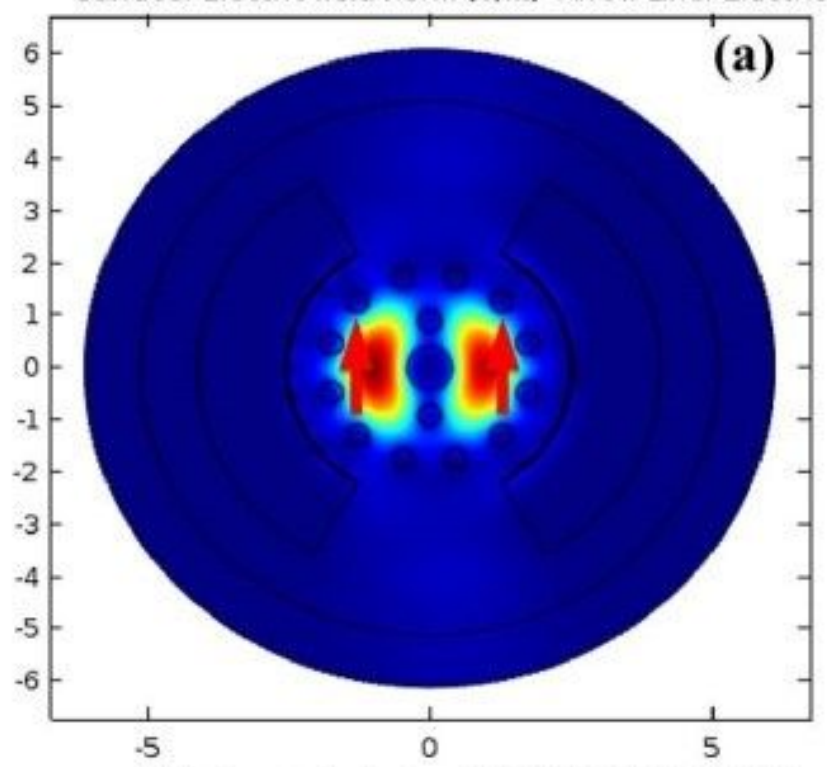

Effective mode index $=1,436066-1.715782 \mathrm{E}-41$ Surface: Electric field norm (V/m) Arrow Line: Electric field

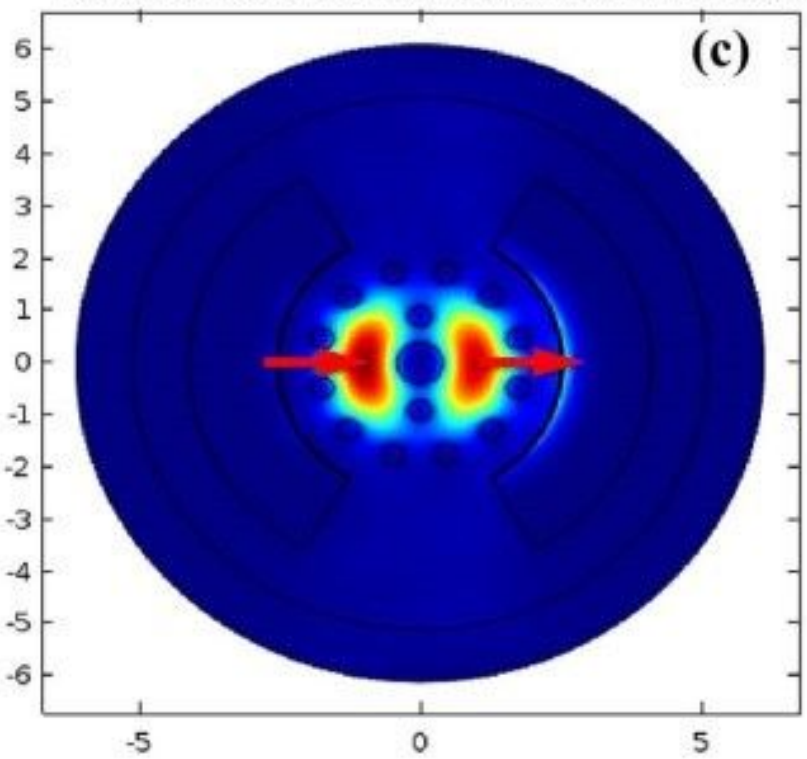

Effective mode index $=1.436364-6.425048 \mathrm{E}-5 \mathrm{i}$ Surface: Electric field norm (V/m) Arrow Line: Electric field

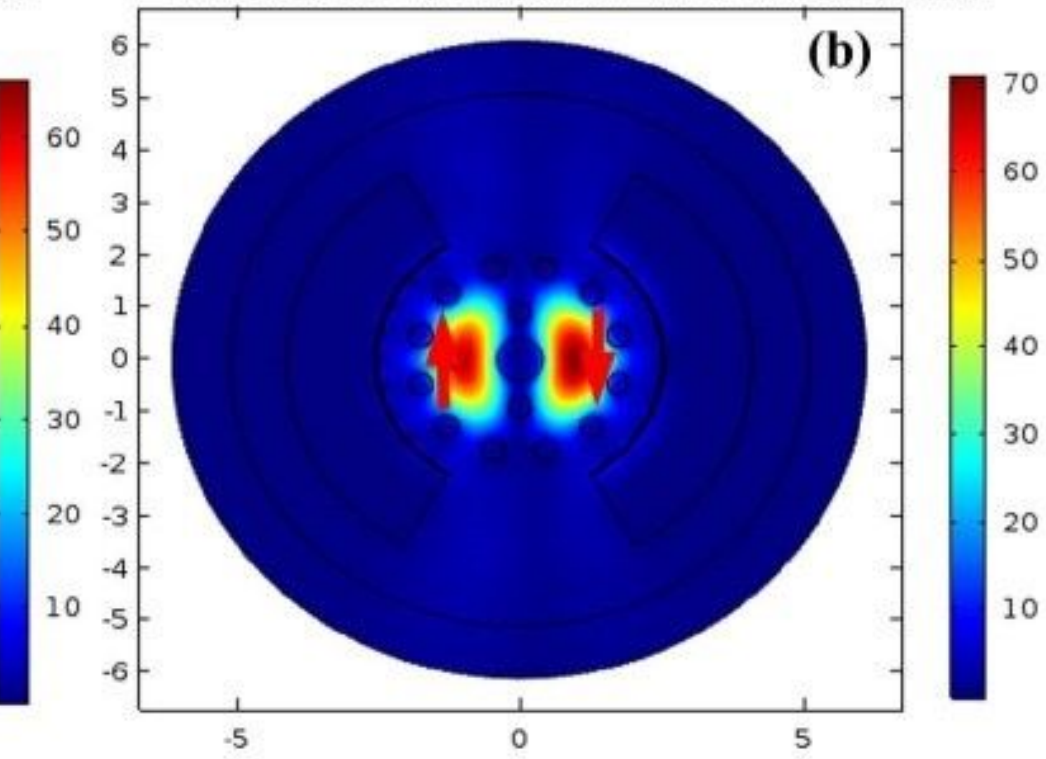

Effective mode index $=1.435465-2.068961 \mathrm{E}-4 \mathrm{i}$ Surface: Electric field norm (V/m) Arrow Line: Electric field

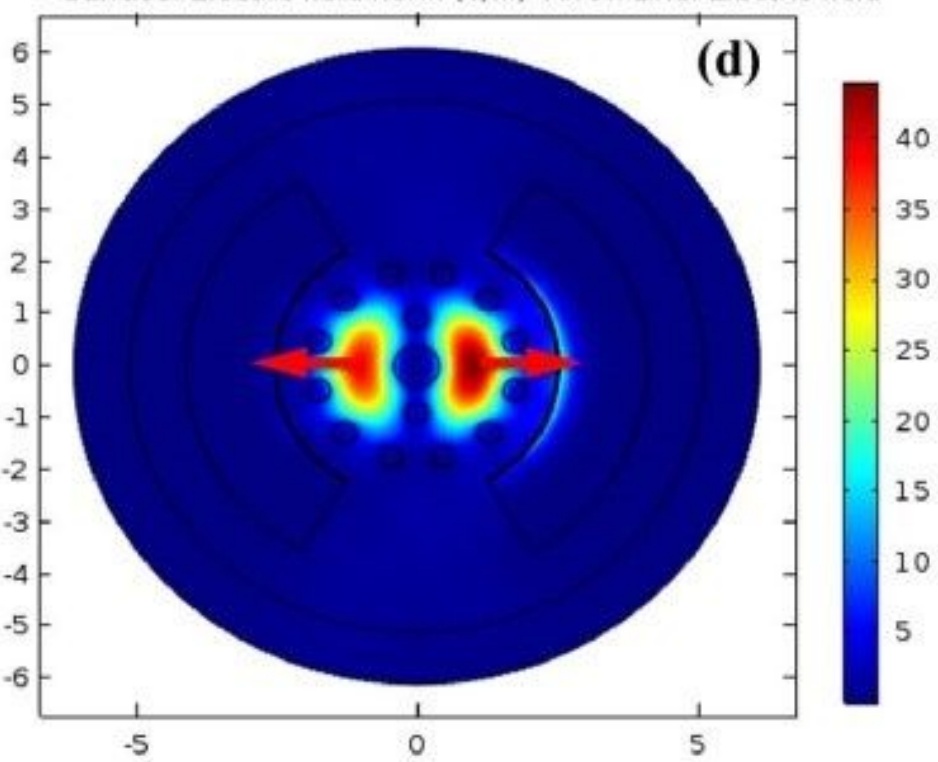

Figure 3

Electric field distribution of the core mode. (a) even mode and (b) odd mode for y-polarization, (c) even mode and (d) odd mode for $x$-polarization. $(\Lambda=0.9 \mu \mathrm{m}, \mathrm{r} 1=0.40 \mu \mathrm{m}, \mathrm{r} 2=0.20 \mu \mathrm{m}, \mathrm{dm}=45 \mathrm{~nm}$, na1=na2=1.340, $\lambda$ peak $=658 \mathrm{~nm}$ ) 


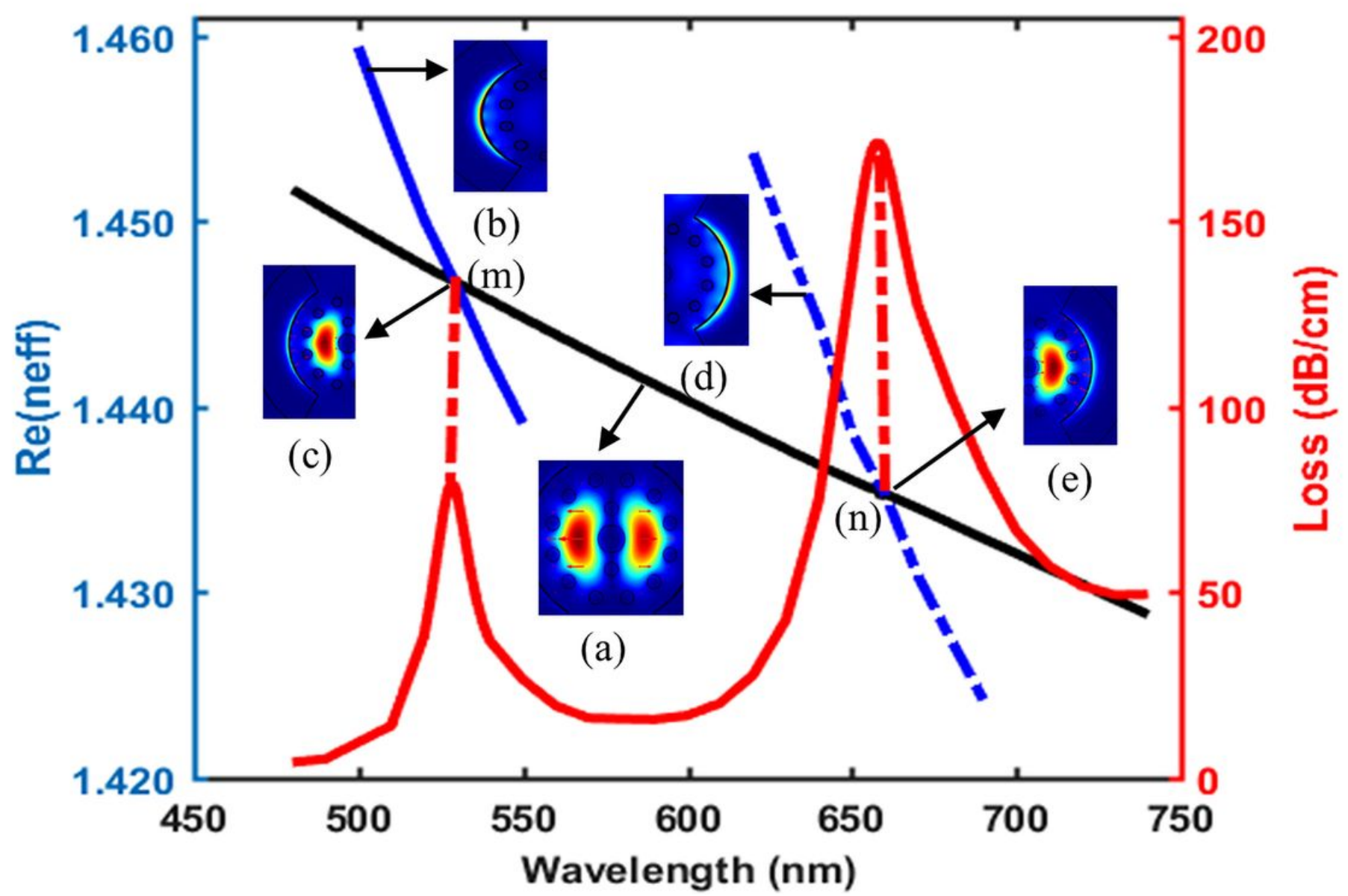

Figure 4

Dispersion relationship of the core mode (black line) and SPR modes (blue solid and dotted lines) and loss spectrum (red line) with insets (a), (b), (c), (d) and (e) showing the electric field distribution. $(\Lambda=0.9$ $\mu \mathrm{m}, \mathrm{r} 1=0.40 \mu \mathrm{m}, \mathrm{r} 2=0.20 \mu \mathrm{m}, \mathrm{dm}=45 \mathrm{~nm}, \mathrm{na} 1=\mathrm{na} 2=1.340$ ) 


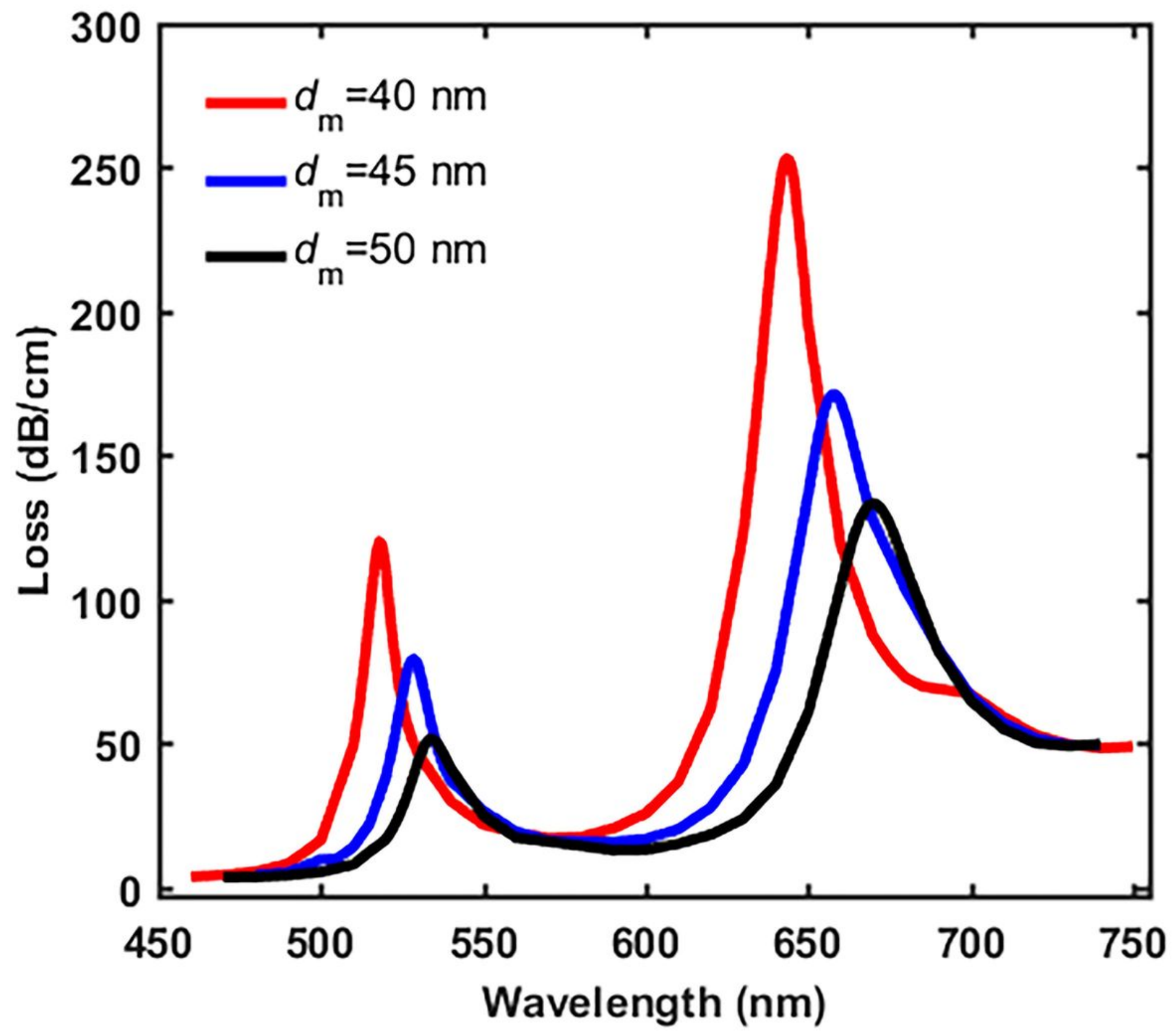

Figure 5

Loss spectrum of the core mode for different $\mathrm{dm}$ from 40 to $50 \mathrm{~nm} .(\Lambda=0.9 \mu \mathrm{m}, \mathrm{r} 1=0.40 \mu \mathrm{m}, \mathrm{r} 2=0.20 \mu \mathrm{m}$, na1=na2=1.340) 


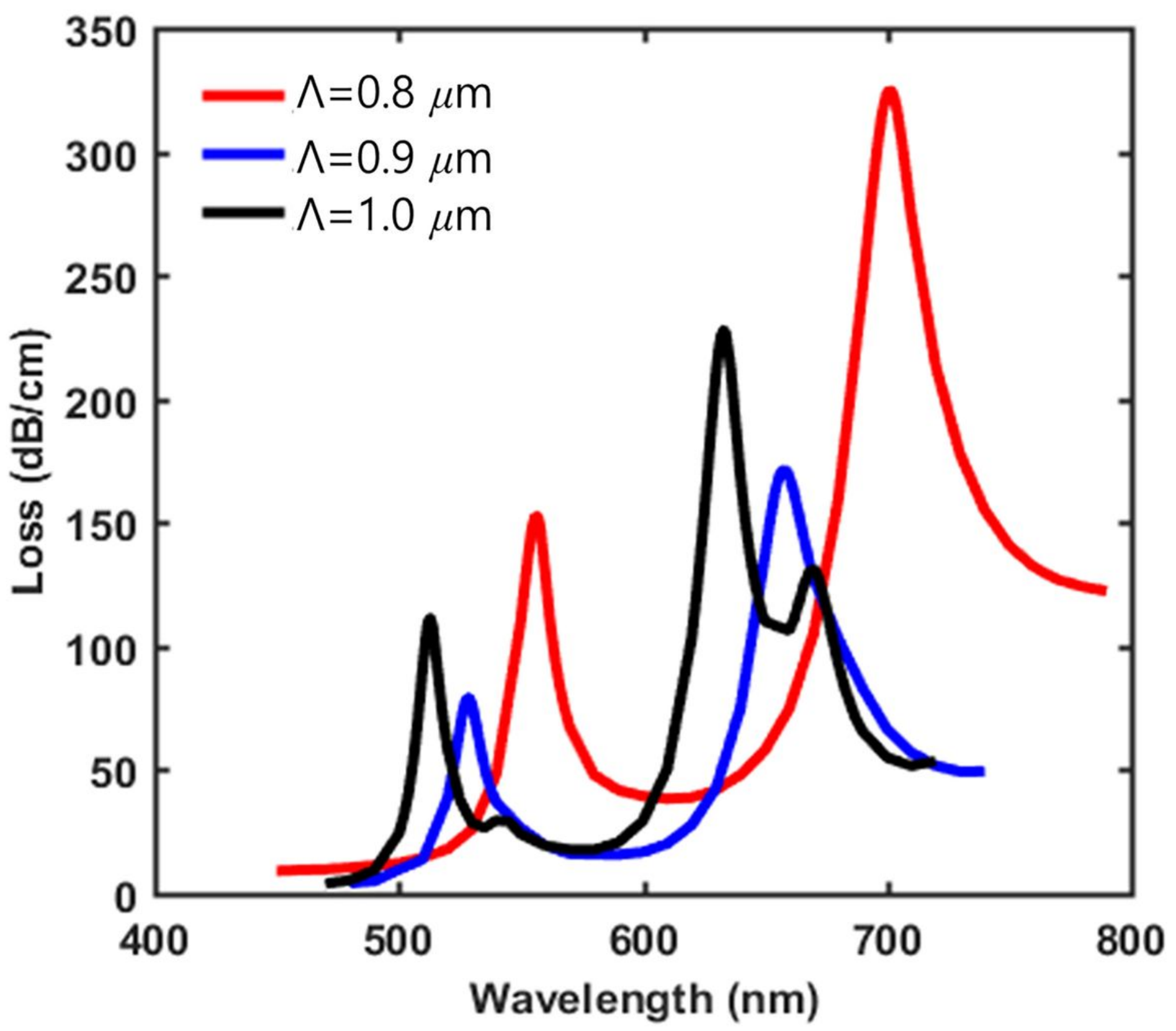

Figure 6

Loss spectrum of the core mode for different $\Lambda$ from 0.8 to $1.0 \mu \mathrm{m}$. $(r 1=0.40 \mu \mathrm{m}, \mathrm{r} 2=0.20 \mu \mathrm{m}, \mathrm{dm}=45 \mathrm{~nm}$, na1 $=$ na2 $=1.340)$ 

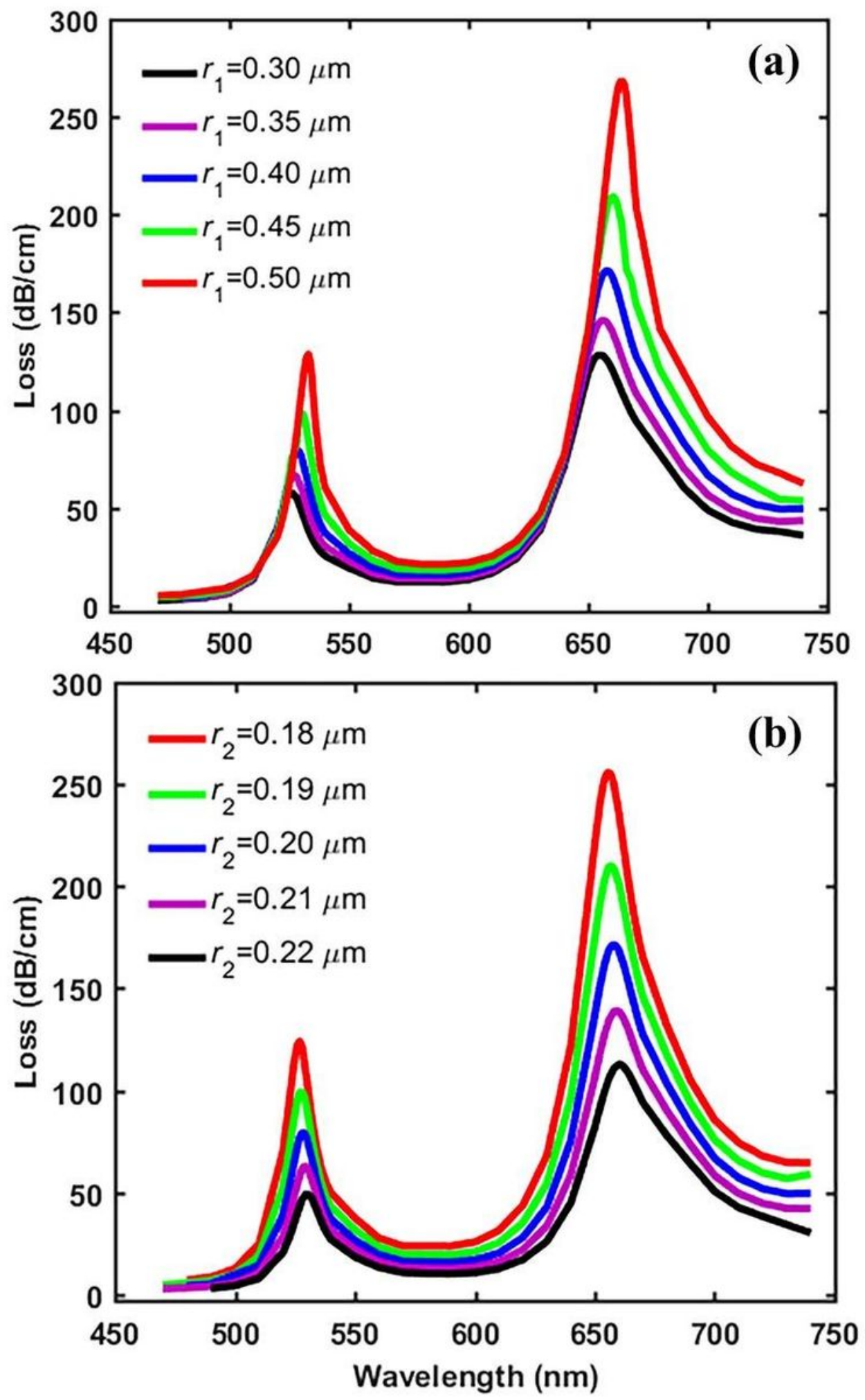

Figure 7

(a) Loss spectrum of the core mode for different $\mathrm{r} 1$ from 0.30 to $0.50 \mu \mathrm{m}$ and (b) different $\mathrm{r} 2$ from 0.18 to $0.22 \mu \mathrm{m} .(\Lambda=0.9 \mu \mathrm{m}, \mathrm{dm}=45 \mathrm{~nm}, \mathrm{na} 1=\mathrm{na} 2=1.340)$ 

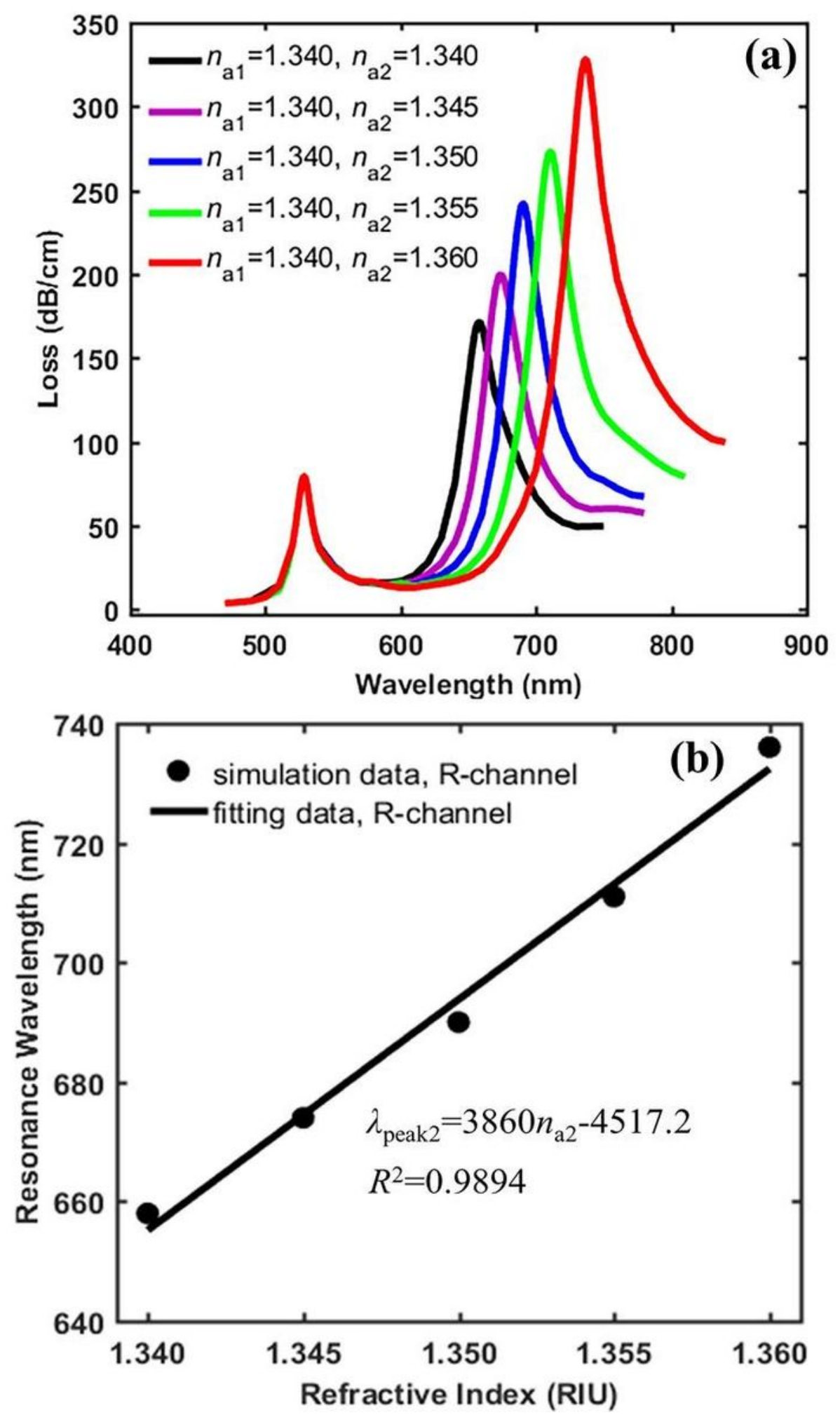

Figure 8

a) Loss spectrum of the core mode for different na2 from 1.340 to 1.360 . (b) Linear fitting line of $\lambda$ peak2 with respect to na2. $(\Lambda=0.9 \mu \mathrm{m}, \mathrm{r} 1=0.40 \mu \mathrm{m}, \mathrm{r} 2=0.20 \mu \mathrm{m}, \mathrm{dm}=45 \mathrm{~nm}, \mathrm{na} 1=1.340)$ 

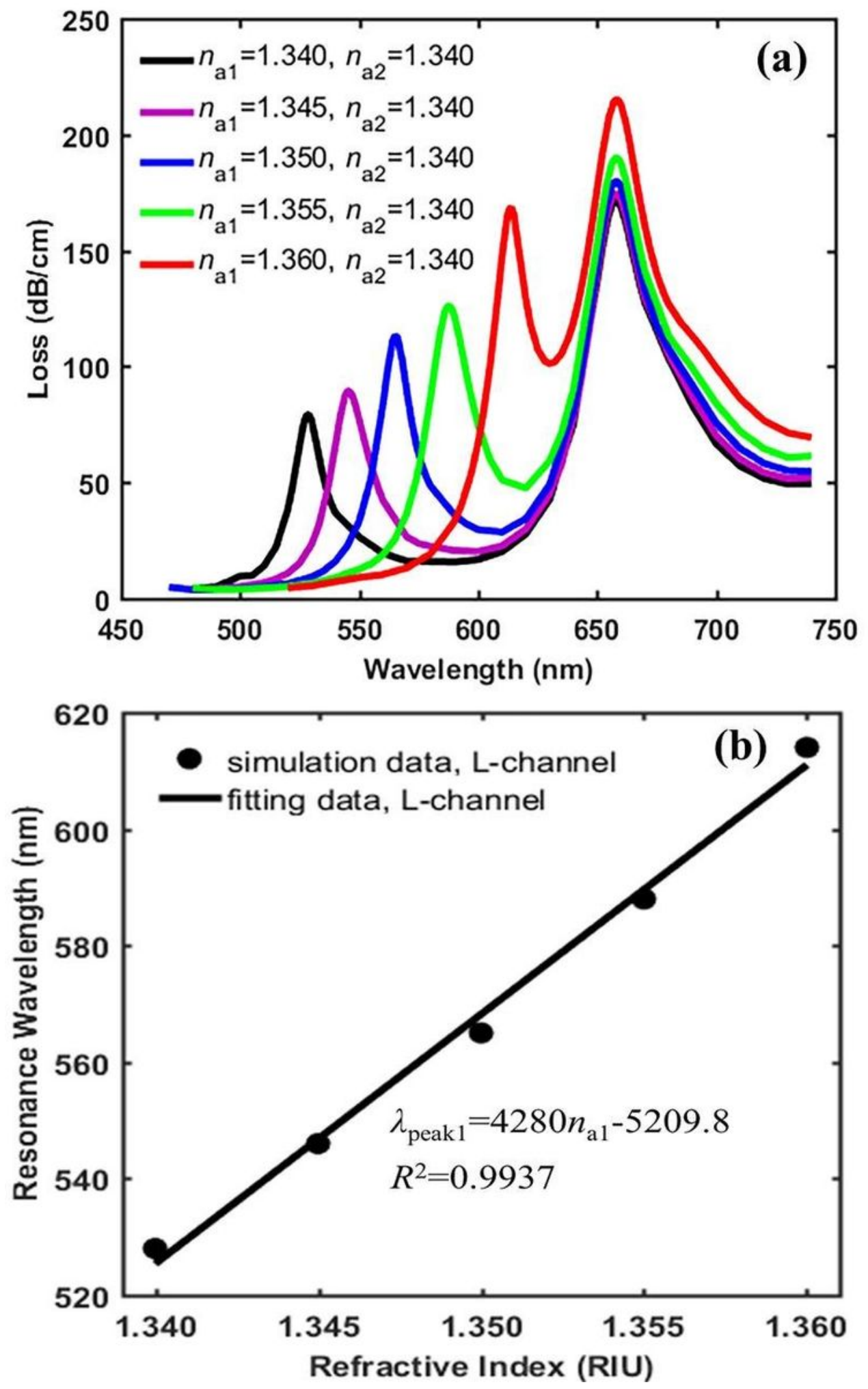

Figure 9

(a) Loss spectrum of the core mode for different na1 from 1.340 to 1.360. (b) Linear fitting line of $\lambda$ peak1 with respect to na1. $(\Lambda=0.9 \mu \mathrm{m}, \mathrm{r} 1=0.40 \mu \mathrm{m}, \mathrm{r} 2=0.20 \mu \mathrm{m}, \mathrm{dm}=45 \mathrm{~nm}, \mathrm{na} 2=1.340)$ 

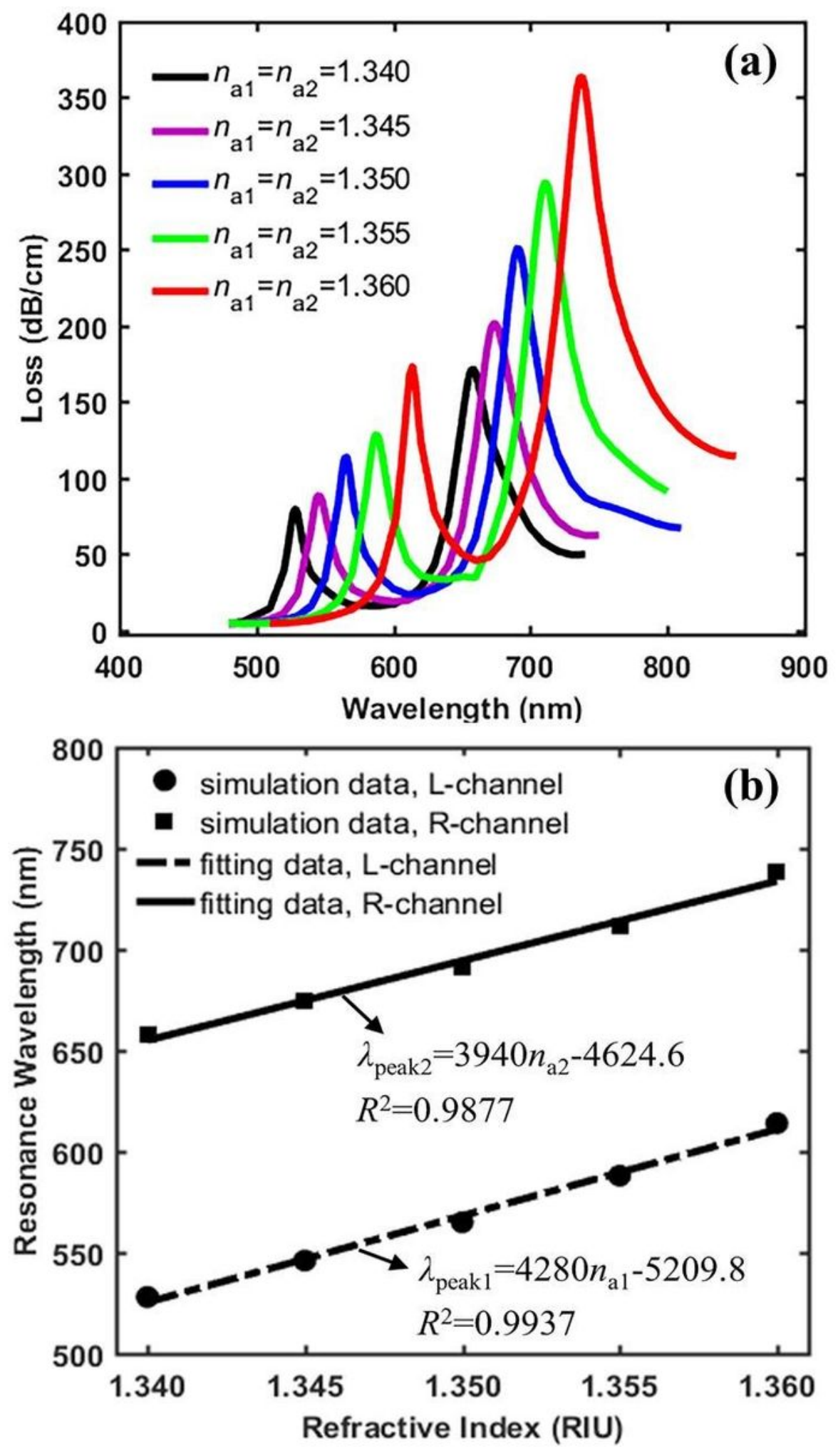

Figure 10

(a) Loss spectrum of the core mode for different na1 and na2 from 1.340 to 1.360 . (b) Linear fitting curves of $\lambda$ peak1 and $\lambda$ peak2 with respect to na1 and na2, respectively. $(\Lambda=0.9 \mu \mathrm{m}, \mathrm{r} 1=0.40 \mu \mathrm{m}, \mathrm{r} 2=0.20$ $\mu \mathrm{m}, \mathrm{dm}=45 \mathrm{~nm}$ ) 